\title{
ARTICLE
}

\section{Imbalance of the reciprocally inhibitory loop between the ubiquitin-specific protease USP43 and EGFR/PI3K/AKT drives breast carcinogenesis}

Lin $\mathrm{He}^{1}$, Xinhua Liu ${ }^{1,2,3}$, Jianguo Yang ${ }^{1}$, Wanjin $\mathrm{Li}^{1}$, Shumeng $\mathrm{Liu}^{2}$, Xujun Liu ${ }^{1}$, Ziran Yang ${ }^{1}$, Jie Ren ${ }^{1}$, Yue Wang ${ }^{2}, \mathrm{Lin} \mathrm{Shan}^{2}$, Chengjian Guan ${ }^{4}$, Fei Pei ${ }^{5}$, Liandi Lei ${ }^{6}$, Yu Zhang ${ }^{1}$, Xia Yi ${ }^{1}$, Xiaohan Yang ${ }^{1}$, Jing Liang ${ }^{1}$, Rong Liu ${ }^{4}$, Luyang Sun ${ }^{1}$ and Yongfeng Shang ${ }^{1,2,3}$

Hyperactivation of EGFR/PI3K/AKT is a prominent feature of various human cancers. Thus, understanding how this molecular cascade is balanced is of great importance. We report here that the ubiquitin-specific protease USP43 is physically associated with the chromatin remodeling NuRD complex and catalyzes H2BK120 deubiquitination. Functionally this coordinates the NuRD complex to repress a cohort of genes, including EGFR, which are critically involved in cell proliferation and carcinogenesis. We show that USP43 strongly suppresses the growth and metastasis of breast cancer in vivo. Interestingly, USP43 also exists in the cytoplasm, where it is phosphorylated by AKT, enabling its binding to the $14-3-3 \beta / \varepsilon$ heterodimer and sequestration in the cytoplasm. Significantly, hyperactivation of EGFR/PI3K/AKT in breast cancer is associated with the cytoplasmic retention of USP43 and thus, the inhibition of its transcriptional regulatory function. Moreover, cancer-associated mutations of USP43 affect its subcellular localization and/or epigenetic regulatory functions. Nuclear USP43 is significantly reduced in breast carcinomas and is associated with EGFR accumulation and AKT hyperactivation. A low level of nuclear USP43 correlates with higher histologic grades and poor prognosis. Our study identifies USP43 to be an H2BK120 deubiquitinase and a potential tumor suppressor and reveals a reciprocally inhibitory loop between USP43 and EGFR/PI3K/AKT, whose imbalance drives breast carcinogenesis.

Cell Research (2018) 28:934-951; https://doi.org/10.1038/s41422-018-0079-6

\section{INTRODUCTION}

It has been well established that epidermal growth factor receptor (EGFR) is required for cell proliferation and animal development and that dysregulation of EGFR is critically involved in malignant transformation and progression of a broad variety of cancers. $^{1-3}$ Binding of EGFR to its cognate ligands leads to the autophosphorylation of receptor tyrosine kinase and subsequent activation of downstream intracellular signaling cascades especially the phosphatidylinositol 3-kinase-AKT serine/threonine kinase 1 (PI3K-AKT) pathway, a molecular axis that is vital for cell proliferation, growth, survival, motility and metabolism. ${ }^{4-6}$ AKT kinase activity is regulated positively by $\mathrm{PI}^{7} \mathrm{~K}^{7}$ and negatively by phosphatase and tensin homolog (PTEN). ${ }^{8}$ Remarkably, gain-offunction mutation/amplification of $E G F R^{9}$ and/or $P I 3 K^{10,11}$ and lossof-function or loss of heterozygosity of PTEN, ${ }^{12}$ which all lead to hyperactivation of AKT, also frequently occur in human cancers. Nevertheless, genetic alterations cannot fully account for the hyperactivation of the EGFR/PI3K/AKT cascade. ${ }^{13}$ It is reported that AKT kinase activity is elevated in approximately $40 \%$ of breast and ovarian cancers and in $>50 \%$ of prostate cancer. Moreover, nearly $80 \%$ of tumors with activated AKT are highergrade carcinomas. ${ }^{14}$ Clearly, understanding the molecular basis, including epigenetic mechanisms, underlying the dysregulation of the EGFR/PI3K/AKT pathway is of great significance.

Ubiquitination is an important cellular process implicated in a multitude of events from endocytosis to transcription and from cell cycle progression to DNA repair. In effect, the covalent attachment of ubiquitin molecules to substrates influences many cellular signaling pathways either through proteasomal degradation or by modulating the activity and/or localization of constituent proteins. Ubiquitination levels are balanced by ubiquitinating enzymes (E1, E2 and E3) and deubiquitinating enzymes (DUBs). ${ }^{15}$ The human genome encodes approximately 98 putative DUBs, which belong to the protease superfamily and are grouped into several subclasses including ubiquitin-specific proteases (USPs), the largest subclass in DUB family with $>50$ members. A hallmark of USPs is the presence of a catalytic core with so-called histidine and cysteine boxes, along with ubiquitinbinding, ubiquitin-like or zinc-finger domains lying N-terminally and/or C-terminally to the catalytic domain. ${ }^{16}$

It has been shown that USPs are involved in a broad spectrum of cellular processes ${ }^{17}$ and implicated in the pathogenesis of various human diseases including cancer. ${ }^{18}$ Several prominent cellular signaling pathways, such as the $\mathrm{p} 53, \mathrm{NFKB}, \mathrm{Wnt}$ and TGF- $\beta$

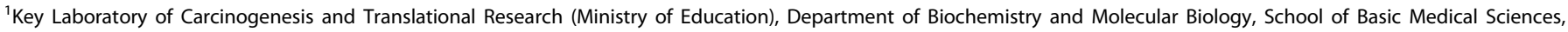

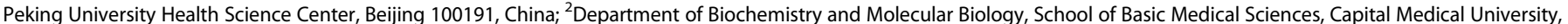

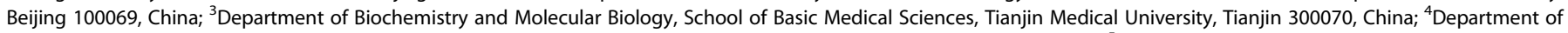

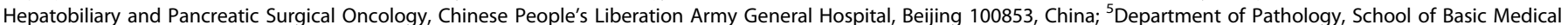

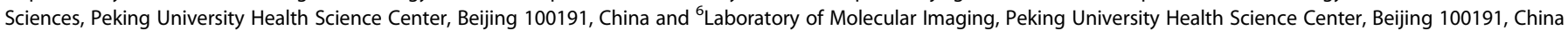
Correspondence: Luyang Sun (luyang_sun@hsc.pku.edu.cn) or Yongfeng Shang (yshang@hsc.pku.edu.cn)
}

Received: 22 March 2018 Revised: 18 June 2018 Accepted: 17 July 2018

Published online: 22 August 2018 
pathways, which are frequently altered in many types of cancer, are profoundly influenced by the activity of various USPs including USP2, USP4, USP5, USP10, USP11, USP15, USP29 and USP34. ${ }^{19}$ In addition, we reported recently that USP7, by influencing several key cell cycle regulators, ${ }^{20}$ and USP9X, by impacting on centrosome duplication, ${ }^{21}$ are involved in breast carcinogenesis. USP9X also regulates mitotic cell death and chemoresistance in aggressive B-cell lymphoma. ${ }^{22}$ Moreover, USP20 and USP33 are implicated in the pathogenesis of Von Hippel-Lindau's (VHL) syndrome. ${ }^{23,24}$ Nevertheless, the enzymatic activity and biological function of the majority of proteins in the USP family including USP43 remain to be determined.

Ubiquitination also occurs on histones, particularly $\mathrm{H} 2 \mathrm{~A}$ and $\mathrm{H} 2 \mathrm{~B} .{ }^{25}$ Mono-ubiquitination of $\mathrm{H} 2 \mathrm{~A}$ and $\mathrm{H} 2 \mathrm{~B}$ represents a type of histone modification that impacts on a range of chromatinassociated events including transcription, DNA replication and chromatin segregation. ${ }^{26}$ Dysregulation of $\mathrm{H} 2 \mathrm{~A} / \mathrm{H} 2 \mathrm{~B}$ ubiquitination underlies several pathological states, particularly cancer. ${ }^{27-29}$ Analogously, histone ubiquitination is balanced by the activities of specific ubiquitin ligases and deubiquitinases. Although addition of H2B120 ubiquitination (H2BK120ub) is mainly catalyzed by the RNF20/40 complex, ${ }^{30}$ removal of this mark has been documented for multiple USP species including USP3, ${ }^{31}$ USP $22,{ }^{32}$ USP12 and USP46, ${ }^{33}$ USP44, ${ }^{18}$ and USP49. ${ }^{34}$ Thus, it becomes important to understand how the enzymatic activities of these USPs are regulated/coordinated and what kind of cellular environment fosters the action of a particular USP in the context of H2BK120ub deubiquitination. Moreover, although it is becoming increasingly clear that different types of histone modification interplay or "crosstalk" to control the epigenetic output, ${ }^{35}$ how histone ubiquitination acts in coordination with other types of histone modification such as histone acetylation remains to be elucidated.

In the current study, we determined the biochemical activity and cellular function of USP43 and explored its mechanistic involvement in breast carcinogenesis. We investigated the molecular basis and clinical/pathological significance of the cellular compartmentalization of USP43 in relation to the EGFR/ $\mathrm{PISK} / \mathrm{AKT}$ axis in the development and progression of breast cancer. This enabled us to identify a reciprocally inhibitory loop between USP43 and EGFR/PI3K/AKT, whose imbalance drives breast carcinogenesis.

\section{RESULTS}

USP43 is physically associated with the NuRD complex and interacts with the $14-3-3 \beta / \varepsilon$ heterodimer

As stated above, recent studies implicate DUBs in the development and progression of various malignancies by influencing multiple key cellular signaling pathways. ${ }^{36,37}$ To further understand the scope and the variety of the mechanistic involvement of DUBs in human cancers, we cloned the gene encoding for USP43 from a human mammary complementary DNA (cDNA) library, a gene that has not been functionally characterized. The cDNA for USP43 (GeneBank NM_153210.4) is 3369 bp in length and contains an open reading frame encoding for a protein of 1123 amino acids. Bioinformatics analysis indicates that USP43 harbors a putative USP domain (Supplementary information, Figure S1a). An amino-acid sequence alignment revealed that human USP43 shares $100 \%$ identity with its homolog in Pan troglodytes, and the similarity of the amino-acid sequence of USP43 with orthologues in other organisms is $82 \%$ in Bos taurus, $79 \%$ in Mus musculus, $79 \%$ in Rattus norvegicus and $50 \%$ in Xenopus tropicalis. Phylogenetic analysis also indicates that USP43 is an evolutionarily well-conserved gene (Supplementary information, Figure S1b).

To explore the cellular function of USP43, we first employed affinity purification and mass spectrometry to interrogate the USP43 interactome in vivo. In these experiments, FLAG-tagged
USP43 (FLAG-USP43) was stably expressed in mammary adenocarcinoma MCF-7 cells. Cellular extracts were subjected to affinity purification using anti-FLAG affinity columns, and the bound proteins were analyzed by mass spectrometry. The results showed that USP43 co-purified with Mi-2 $\beta$, MTA3, HDAC1/2, RbAp46/48 and MBD3, all components of the nucleosome remodeling and deacetylase (NuRD) complex, as well as with several other proteins including $14-3-3 \beta$ and $14-3-3 \varepsilon$ (Fig. $1 \mathrm{a}$ ), members of the $14-3-3$ family of adaptors that are primarily localized in the cytoplasm and bind to client proteins as homo- or hetero-dimers in a phosphoserine/threonine motif-dependent manner. ${ }^{38}$ The presence of the NuRD subunits and 14-3-3 species in the USP43 interactome was confirmed by western blotting of the columnbound proteins with antibodies against the corresponding putative partner proteins (Fig. 1b). The detailed results of the mass spectrometric analysis are provided in the Supplementary information, Table S1.

To verify the in vivo interaction of USP43 with the NuRD complex and 14-3-3 proteins, total proteins from MCF-7 or MDAMB-231 cells were extracted for immunoprecipitation (IP) experiments using antibodies detecting the endogenous proteins. IP with commercial polyclonal antibodies against USP43 followed by immunoblotting (IB) with antibodies against Mi-2 $\beta$, MTA3, HDAC1, HDAC2, RbAp46/48, MBD3, $14-3-3 \beta$ or $14-3-3 \varepsilon$ demonstrated that each of these tested NuRD components and also 14-3-3 $\beta$ and 14$3-3 \varepsilon$ efficiently co-IP with USP43 from extracts of breast cancer cell lines with low or high metastatic potential (Fig. 1c).

To understand the molecular interaction between USP43 and the NuRD complex, glutathione S-transferase (GST) pull-down assays were performed with GST-fused USP43 (GST-USP43) and individual components of the NuRD complex synthesized by coupled transcription/translation in vitro. These experiments revealed that USP43 was capable of interacting with MTA3 and HDAC1, but not with the other components of the NuRD complex that we tested (Fig. 1d), thus suggesting that the association of USP43 with the NuRD complex is through interactions with MTA3 and HDAC1. Analogously, GST pull-down assays with GST-14-3-3ع and USP43 or $14-3-3 \beta$ synthesized in vitro by coupled transcription/translation showed that USP43 was able to interact with the $14-3-3 \beta / \varepsilon$ heterodimer (Fig. $1 \mathrm{~d}$ ).

To further support the physical interaction of USP43 with the NuRD complex and $14-3-3 \beta / \varepsilon$ in vivo, cellular proteins extracted in high salt from MCF-7 cells were fractionated by size exclusion using fast protein liquid chromatography (FPLC) with Superose 6 columns. We found that native USP43 from MCF-7 cell extracts was eluted with an apparent molecular mass much greater than that of the monomeric protein; USP43 immunoreactivity was detected in chromatographic fractions with an elution pattern that largely overlapped with the NuRD proteins Mi-2 $\beta$, MTA3, HDAC1/2, RbAp46/48 and MBD3 and at least partially overlapped with that of $14-3-3 \beta / \varepsilon$ (Fig. 1e). Importantly, analysis of the FLAG-USP43 affinity eluate from FPLC after Superose 6 gel filtration revealed that USP43 existed in multiprotein complexes; one complex peaked in fraction 14 containing Mi-2 $\beta, \mathrm{MTA} 3, \mathrm{HDAC} 1 / 2, \mathrm{RbAp} 46 / 48$ and MBD3, and another peaked in fraction 30 containing $14-3-3 \beta / \varepsilon$ and HSP71/72 (Supplementary information, Figure S2). Collectively, these experiments support the observation that USP43 is physically associated with the NuRD complex and interacts with the $14-3-3 \beta / \varepsilon$ heterodimer in vivo in a transient yet likely functionally relevant manner.

Phosphorylation of USP43 by AKT leads it to bind to the $14-3-3 \beta / \varepsilon$ heterodimer and be sequestered in the cytoplasm

As stated above, $14-3-3 \mathrm{~s}$ are adaptor proteins that are primarily localized in the cytoplasm, ${ }^{38}$ whereas NuRD is a chromatin remodeling complex existing in the nucleus. ${ }^{39-41}$ Thus, the physical interactions of USP43 with $14-3-3 \beta / \varepsilon$ and the NuRD 
complex imply that USP43 might be compartmentalized in both the cytoplasm and nucleus. Indeed, immunofluorescent staining of USP43 in MCF-7 cells showed that USP43 was found in both compartments (Fig. 2a). In addition, IP of cytoplasmic and nuclear extracts of MCF-7 cells using antibodies directed against USP43 followed by IB with antibodies against $14-3-3 \beta / \varepsilon$ or MTA3 demonstrated that USP43 was mainly associated with $14-3-3 \beta / \varepsilon$ in the cytoplasm while with MTA3 in the nucleus (Fig. 2b). This was confirmed using a DuoLink proximity ligation assay in MCF-7 cells with anti-USP43, anti-MTA3 or anti-14-3-3 $\beta$ antibodies (Fig. 2b).

To search for the involvement of serine/threonine phosphorylation in the interaction with the 14-3-3 adaptors, ${ }^{38}$ we carried out IP
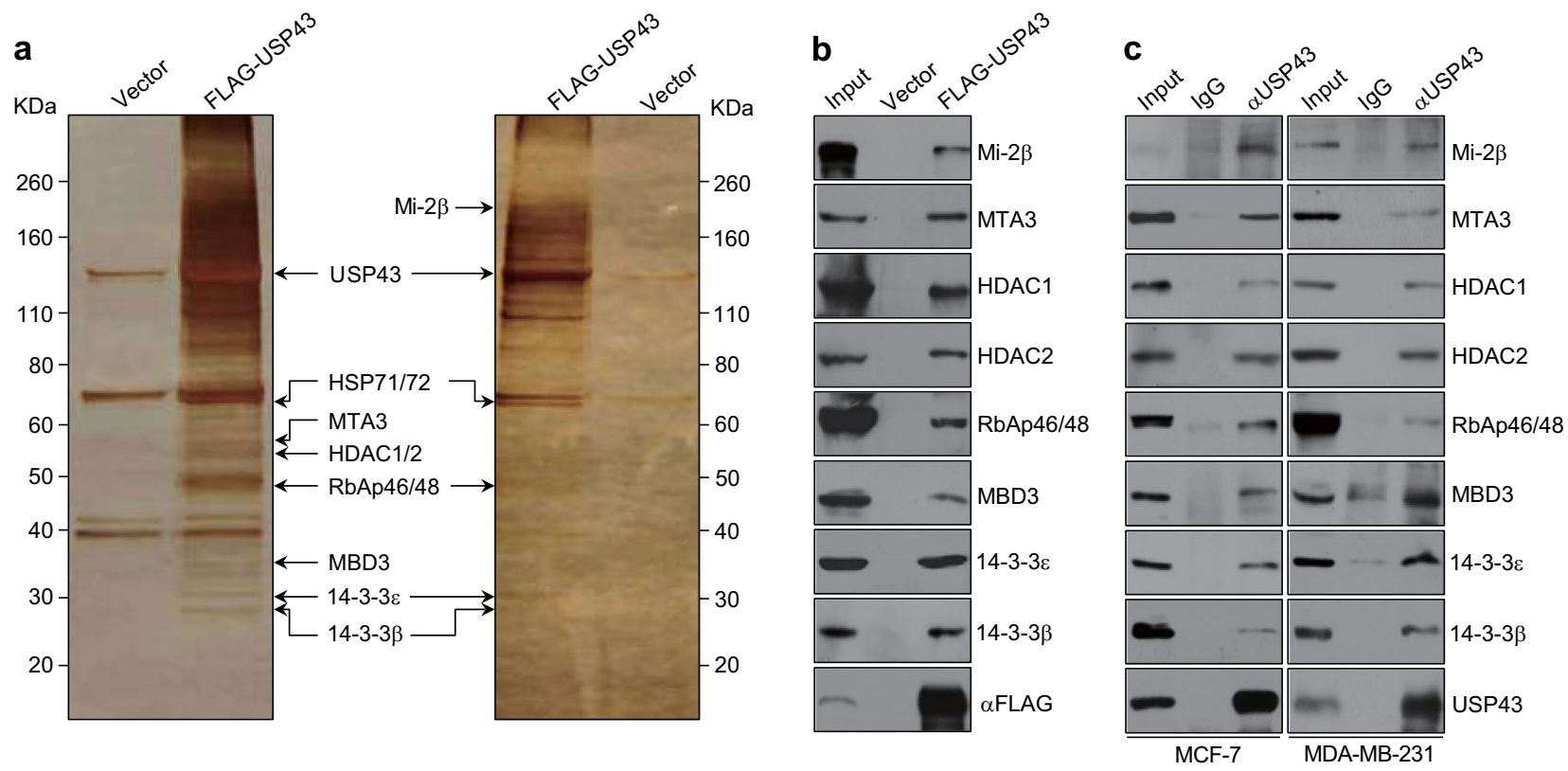

d

e
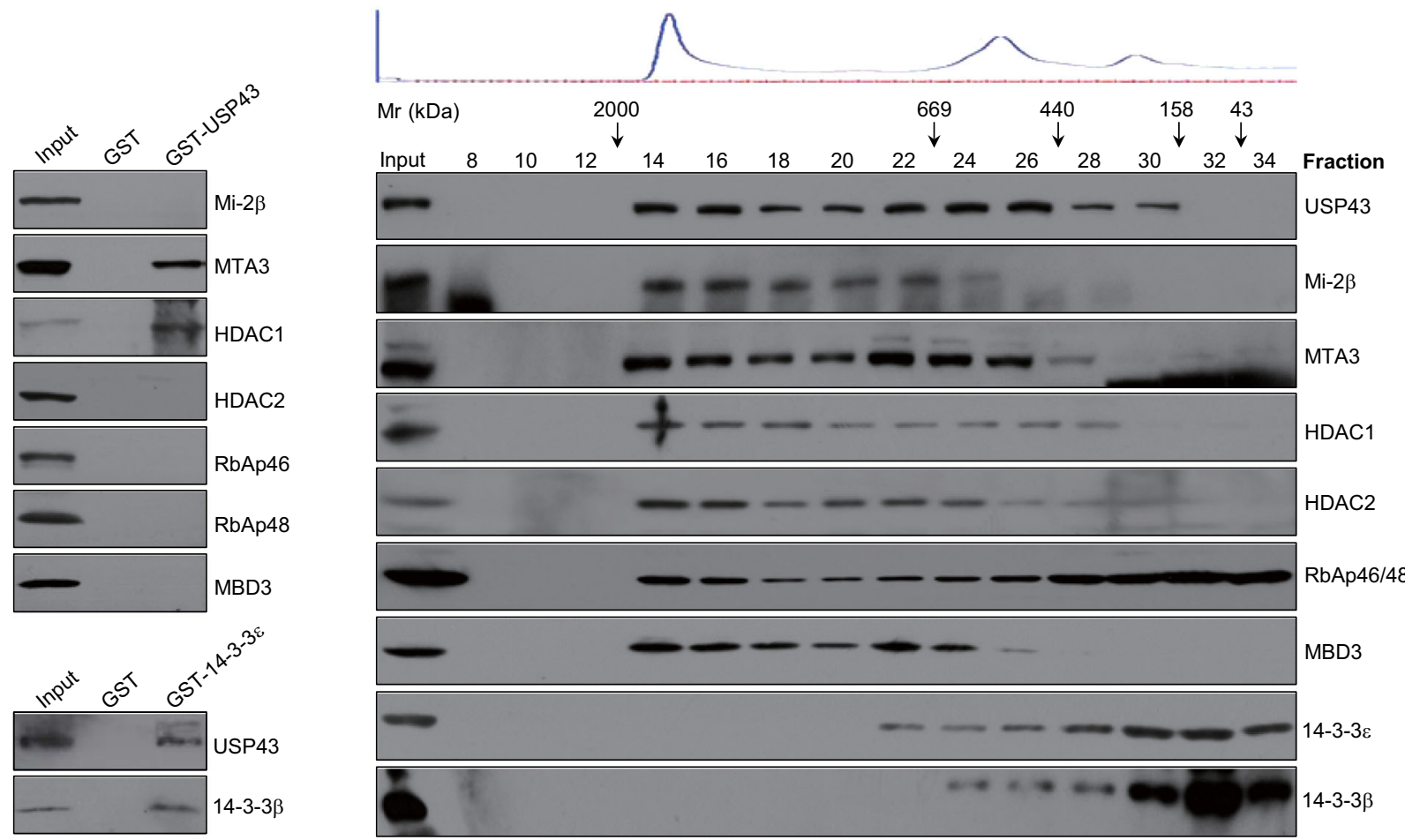

Fig. 1 USP43 is physically associated with the NuRD complex and interacts with $14-3-3 \beta / \varepsilon$ heterodimer. a Cellular extracts from MCF-7 cells stably expressing FLAG-USP43 were subjected to affinity purification with anti-FLAG affinity columns and eluted with FLAG peptides. The eluates were resolved by SDS-PAGE and silver stained. The protein bands were retrieved and analyzed by mass spectrometry. $\mathbf{b}$ Columnbound proteins were analyzed by western blotting using antibodies against the indicated proteins. c Co-immunoprecipitation in MCF-7 or MDA-MB-231 cells with anti-USP43 followed by immunoblotting with antibodies against the indicated proteins. d GST pull-down assays with GST-fused USP43 or 14-3-3 $\varepsilon$ and in vitro transcribed/translated proteins as indicated. e MCF-7 cell proteins were extracted, concentrated and fractionated on Superose 6 size exclusion columns. Chromatographic eluate profiles and the eluate positions of calibration proteins with known molecular masses $(\mathrm{kDa})$ are indicated. An equal volume from each chromatographic fraction was analyzed by western blotting 


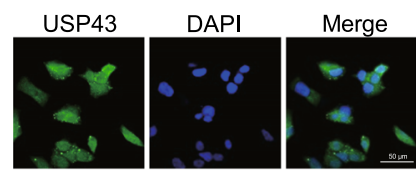

C

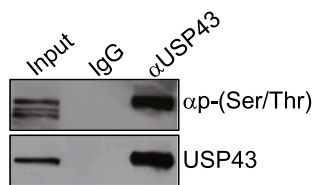

d

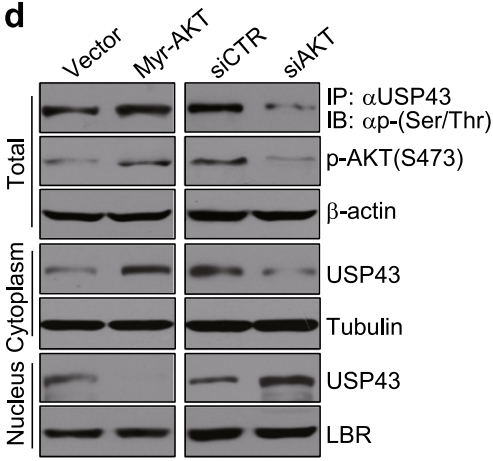

f
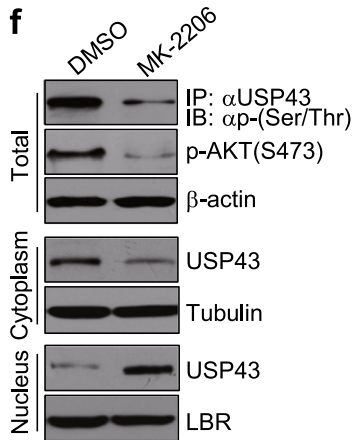

b
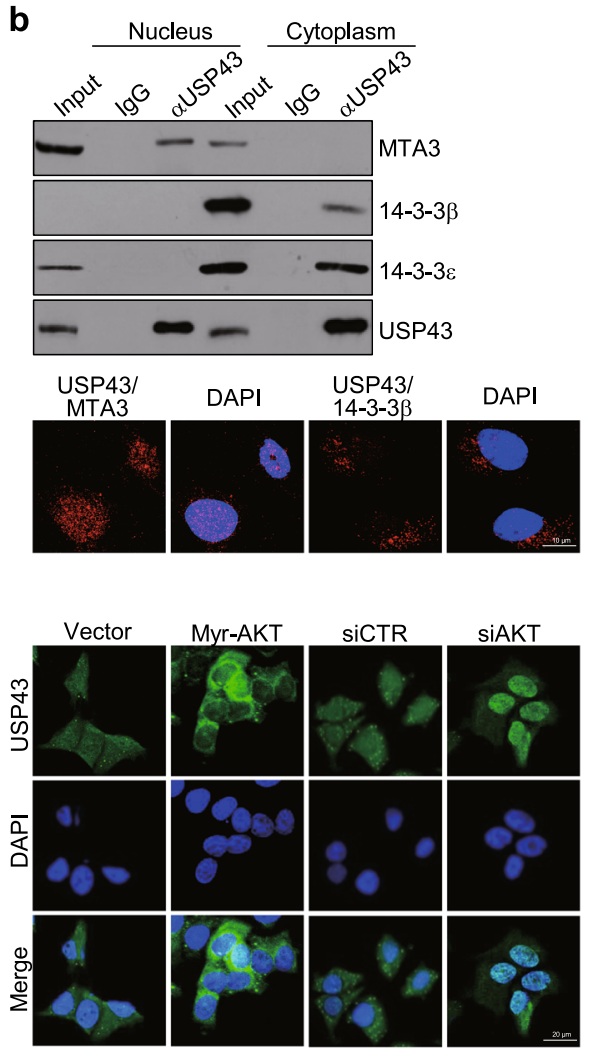

e
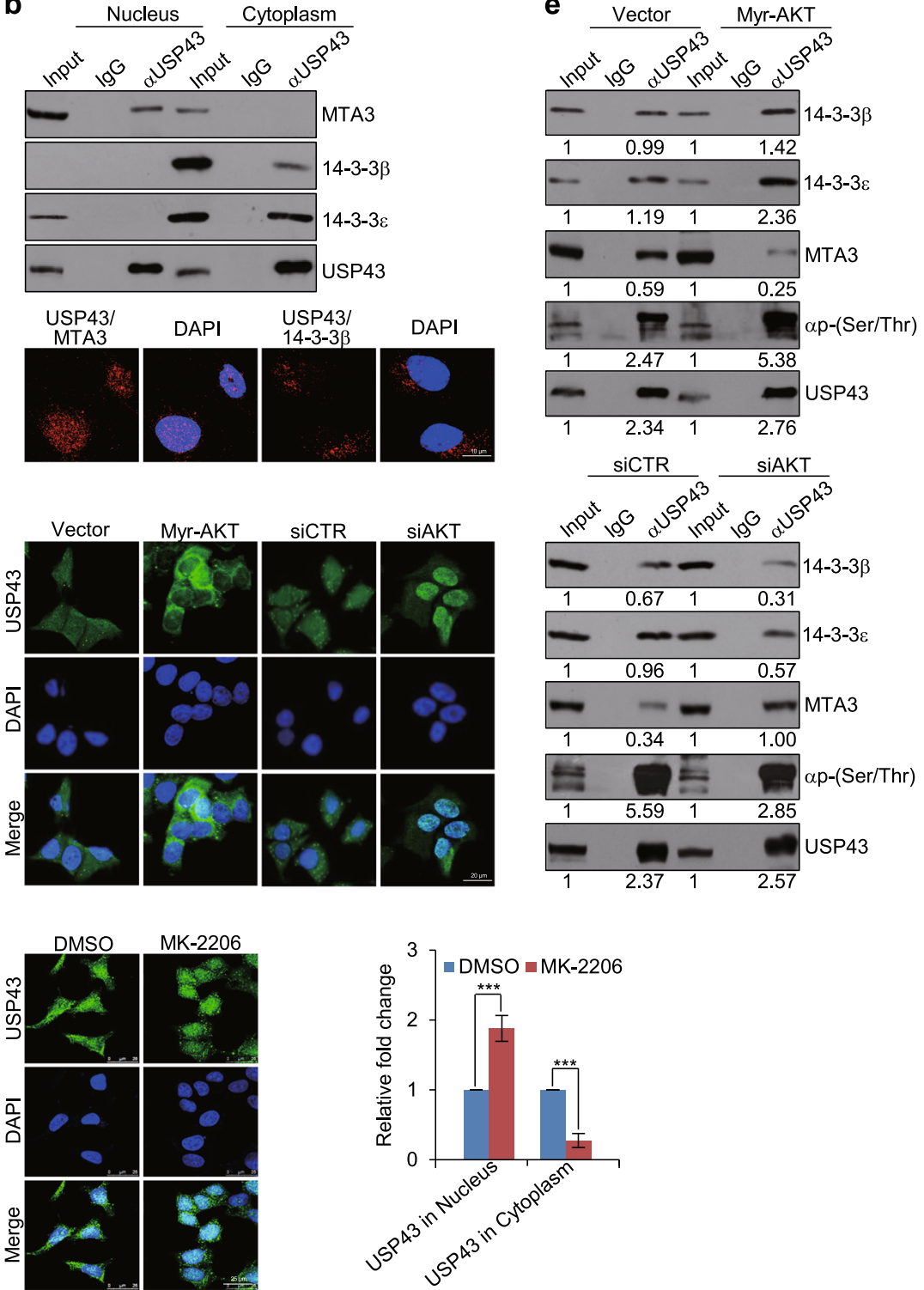

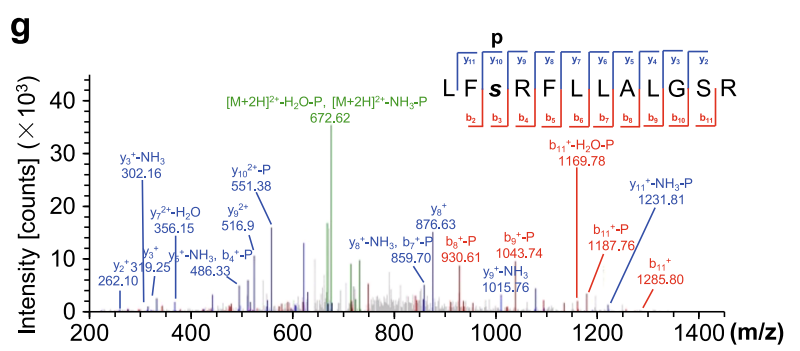

of MCF-7 cell lysates with antibodies against USP43 followed by IB with antibodies against phospho-(Ser/Thr). This clearly indicated that USP43 is a phosphoprotein (Fig. 2c). In this regard, it is interesting to note that a recent stable isotope labeling by amino acids in cell culture (SILAC)-based quantitative phosphoproteomic study upon a human protein microarray-based AKT kinase assay platform showed that aberrant activation of PI3K pathway leads to an increased phosphorylation of a number of proteins including CTTN, TRIP10, PRKCD and USP43 in PIK3CA-mutated cancer cells. ${ }^{11}$ Thus, it is possible that phosphorylation of USP43 by AKT regulates the interaction of USP43 with the $14-3-3 \beta / \varepsilon$ heterodimer. To test this, we overexpressed constitutively active $A K T^{42}$ or knocked down endogenous AKT in MCF-7 cells and examined the phosphorylation status and the subcellular localization of USP43 by IP/IB or immunofluorescent staining. We found that overexpression of constitutively active AKT (Myr-AKT) was associated with increased phosphorylation of USP43, accompanied by its increased cytoplasmic localization and decreased nuclear abundance. In contrast, knockdown of AKT was associated with decreased phosphorylation of USP43, its decreased cytoplasmic 
Fig. 2 Phosphorylation of USP43 by AKT leads to its binding to $14-3-3 \beta / \varepsilon$ heterodimer and sequestration in cytoplasm. a The endogenous USP43 in MCF-7 cells was immunofluorescently stained with polyclonal antibodies against USP43. DAPI staining was included to visualize the nucleus (blue). b Co-immunoprecipitations of nuclear or cytoplasmic proteins in MCF-7 cells with anti-USP43 followed by immunoblotting with antibodies against the indicated proteins (upper). Duolink proximity ligation assay in MCF-7 cells was performed with anti-USP43, antiMTA3 or anti-14-3-3 $\beta$ antibodies (lower). c Co-immunoprecipitations in MCF-7 cells with anti-USP43 followed by immunoblotting with antiphospho-(Ser/Thr) or anti-USP43. d MCF-7 cells were transfected with constitutively active AKT plasmids (Myr-AKT) or AKT siRNAs prior to nucleus-cytoplasm separation for western blot analysis of USP43 expression and the level of AKT phosphorylation (left). The subcellular localization of USP43 in MCF-7 cells transfected with Myr-AKT plasmids or treated with AKT siRNAs was analyzed by immunofluorescent staining (right). e Co-immunoprecipitation assays in MCF-7 cells upon overexpression or knockdown of AKT with anti-USP43 followed by immunoblotting with the indicated antibodies. The bands were quantified with ImageJ software. The numbers indicate the relative levels of the indicated proteins or modifications. f MCF-7 cells were treated with DMSO or AKT inhibitor prior to nucleus-cytoplasm separation for western blot analysis of USP43 expression and the level of AKT phosphorylation (left). The subcellular localization of USP43 in MCF-7 cells treated with DMSO or AKT inhibitor was analyzed by immunofluorescent staining and the quantification is shown (right). The data presented are mean \pm SD for three independent experiments $\left({ }^{* *} p<0.001\right)$. $g$ In vitro phosphorylation assays with bacterially expressed GST-USP43 and Sf9 cells-expressed AKT followed by LC-MS/MS. The relevant ion fragments are labeled, and the corresponding peptide positions are illustrated. $\mathrm{b}$ and $\mathrm{y}$ represent the cleavage points of the peptide backbone when the charge is retained at the $\mathrm{N}$ - or $\mathrm{C}$-terminal fragments of the peptide, respectively

localization and increased nuclear abundance (Fig. 2d). In addition, IP of USP43 from MCF-7 cells followed by IB with antibodies against $14-3-3 \beta, 14-3-3 \varepsilon$ or MTA3 showed that overexpression of constitutively active AKT was associated with enhanced interaction of USP43 with the $14-3-3 \beta / \varepsilon$ heterodimer and a weakened interaction with MTA3. In contrast, AKT depletion resulted in a weakened interaction of USP43 with the $14-3-3 \beta / \varepsilon$ heterodimer and enhanced interaction with MTA3 (Fig. 2e). Together, these experiments strongly suggest that AKT-mediated USP43 phosphorylation triggers 14-3-3 binding and thus, the cytoplasmic retention of USP43.

To gain further support for this notion, MCF-7 cells were treated with MK-2206, an allosteric AKT inhibitor. ${ }^{43}$ Western blot analysis of the cytoplasmic and nuclear extracts of these cells showed that inhibition of AKT activity resulted in a decreased phosphorylation and cytoplasmic localization of USP43, accompanied by an increased level of USP43 in the nucleus (Fig. 2f). In agreement, immunofluorescent staining of USP43 in these cells showed that inhibition of AKT activity led to decreased cytoplasmic localization and increased nuclear abundance of this protein (Fig. 2f). Furthermore, the in vitro phosphorylation of bacterially expressed GST-USP43 by AKT expressed in Sf9 cells followed by liquid chromatography/tandem mass spectrometry (LC-MS/MS) demonstrated that AKT could phosphorylate USP43 upon Ser29 (Fig. 2g). To validate a requirement for phosphorylation of USP43 at Ser29 to its cytoplasmic retention by the $14-3-3 \beta / \varepsilon$ heterodimer, we transfected MCF-7 cells with Myr-AKT and/or USP43/S29A plasmids or treated the cells with 14-3-3 $\beta$ small interfering RNA (siRNA) before separating nucleus and cytoplasm fractions for western blot analysis of USP43 expression. The results showed that either depletion of $14-3-3 \beta$ or overexpression of USP43/S29A abolished the AKT-mediated cytoplasmic retention of USP43 (Supplementary information, Figure S3). Collectively, the above experiments support the notion that USP43 is phosphorylated by AKT at Ser29, leading to its binding to the $14-3-3 \beta / \varepsilon$ heterodimer and sequestration in the cytoplasm.

USP43 is a deubiquitinase acting on H2BK120 ubiquitination To understand the functional significance of the physical interaction of USP43 with the NuRD complex and to explore the potential deubiquitinase activity of USP43, we first investigated whether or not USP43 could promote deubiquitination to enhance the stability of MTA3 and HDAC1, subunits of the NuRD complex that were identified to interact with USP43 directly. Western blot analysis showed that neither overexpression nor knockdown of USP43 affected the protein levels of MTA3 and HDAC1 (Fig. 3a) arguing against these proteins being targeted for deubiquitination/stabilization by USP43.
The NuRD complex is empowered with both ATP-dependent chromatin remodeling and histone deacetylase activities and functions in epigenetic regulation. ${ }^{39,40}$ The interaction of USP43 with this complex suggests that USP43 might be functionally linked to epigenetic regulation, particularly histone ubiquitination. To test this hypothesis, we utilized the SILAC-LCMS/MS approach to quantify dynamic changes in the level of histone ubiquitination in vivo. In these experiments, MCF-7 cells were infected with empty lentiviral vectors or vectors carrying a USP43 expression construct and maintained in "light" $\left[{ }^{12} \mathrm{C}_{6}\right]-\mathrm{L}-$ lysine- or "heavy" $\left[{ }^{13} \mathrm{C}_{6}\right]$-L-lysine-containing medium, respectively. Histones were acid extracted from chromatin, trypsin-digested and enriched using anti-di-Gly-conjugated agarose beads for LCMS/MS analysis (Fig. 3b). The signal intensity from light and heavy peptides provides a quantitative comparison of their relative abundances. We were able to detect multiple ubiquitinated lysine sites on histones, including H2BK120, H3K56, H4K31, H2BK108, H2AK118 and H2AK119 (Table 1). Among these histone ubiquitinations, the levels of H2BK120ub, H3K56ub and H4K31ub were significantly reduced upon USP43 overexpression, whereas the levels of H2BK108ub, H2AK118ub and H2AK119ub were essentially unaffected (Fig. 3c). Specifically, LC-MS/MS detected a 2.17fold decrease in H2BK120ub in cells overexpressing USP43 (Fig. 3d), suggesting that $\mathrm{H} 2 \mathrm{BK} 120 \mathrm{ub}$ is at least one of the targets for deubiquitination by USP43.

As stated above, several DUBs have already been identified as deubiquitinases for H2BK120ub. ${ }^{18,31-34}$ To gain further support for the deubiquitination of H2BK120ub by USP43 and to have a global view of H2BK120ub deubiquitination by DUBs, we carried out a systematic screen to knockdown each of 81 DUBs using an siRNA library containing a pool of four different siRNAs against each target and assaying for H2BK120ub deubiquitination. High-content screening (HCS) verified the known deubiquitination of H2BK120ub by DUBs USP12 and USP22 (Fig. 3e). These experiments also showed that the average immunofluorescent intensity of H2BK120ub significantly increased upon USP43 depletion (Fig. 3e), supporting its role in H2BK120ub deubiquitination. In addition, treatment of HeLa cells with siRNA pools specific for USP12, USP22, USP43 or USP30 and western blot analysis of acid extracted histones with anti-H2BK120ub showed that the level of H2BK120ub increased upon knockdown of either USP12, USP22 or USP43, whereas depletion of USP30 had limited effect (Fig. 3f). Moreover, western blotting showed that overexpression of USP43 in U2OS and HeLa cells resulted in a decrease in H2BK120ub level whereas knockdown of USP43 in HEK293T and MCF-7 cells led to increased H2BK120ub levels (Fig. 3g). Together, these results support the role of USP43 in H2BK120ub deubiquitination. 

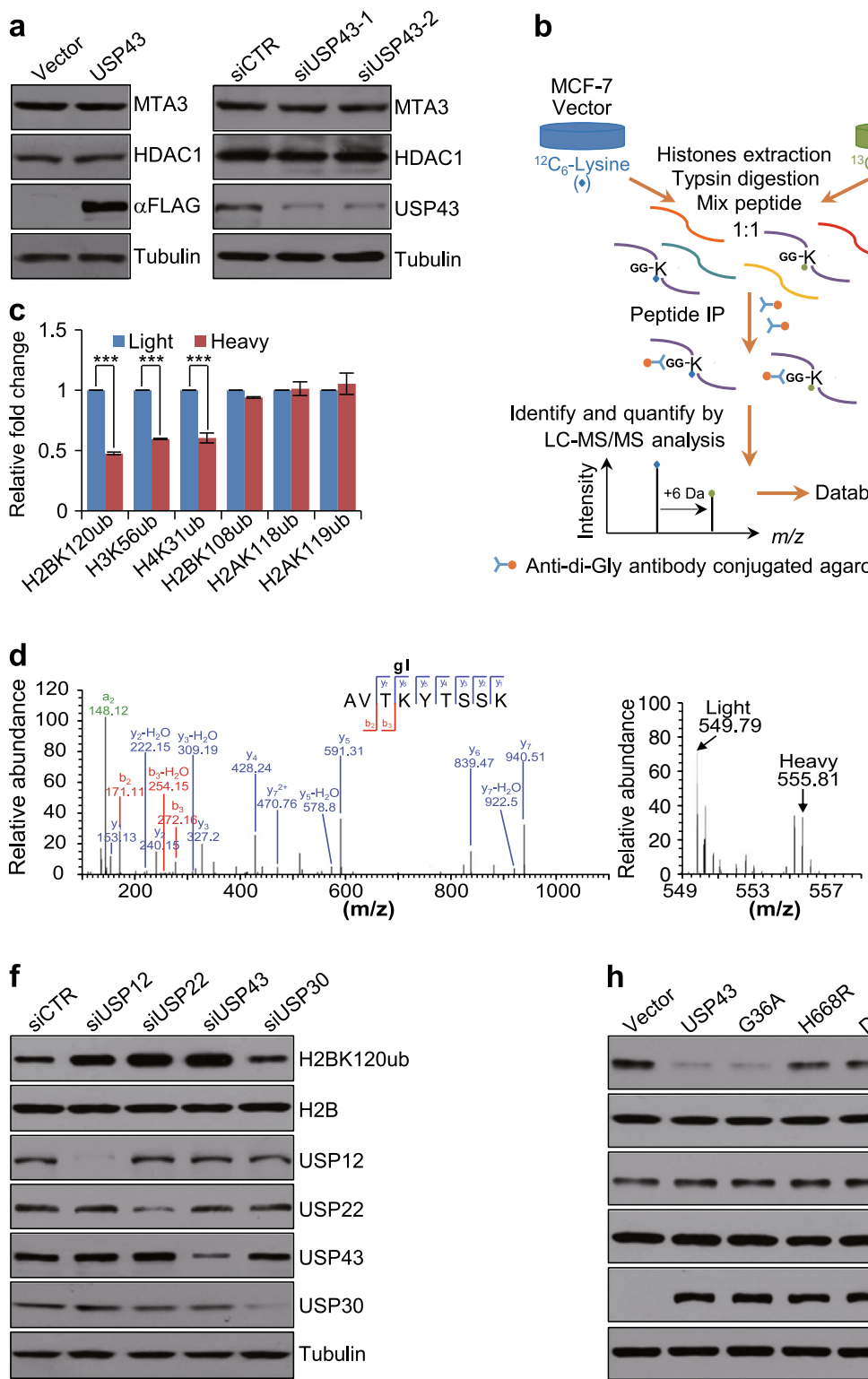

h

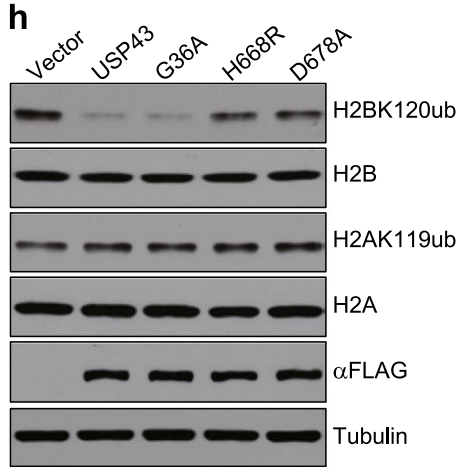

e
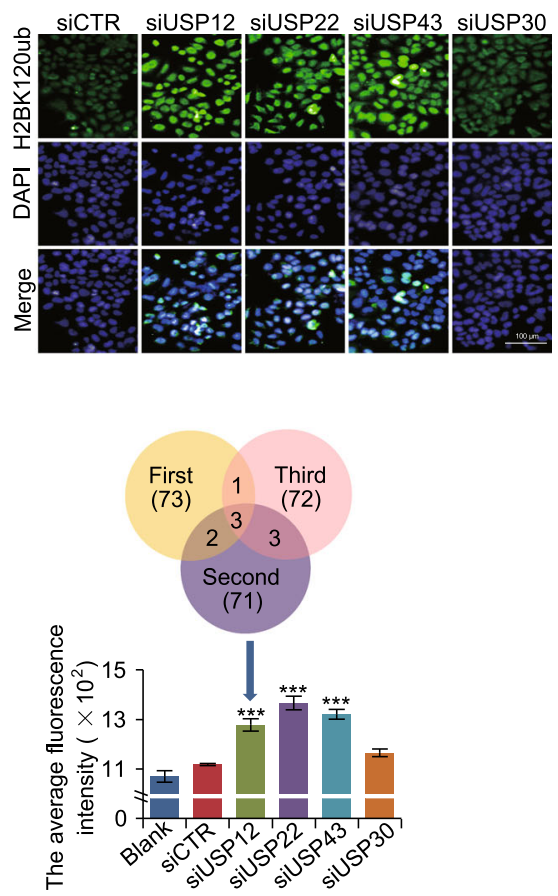

g

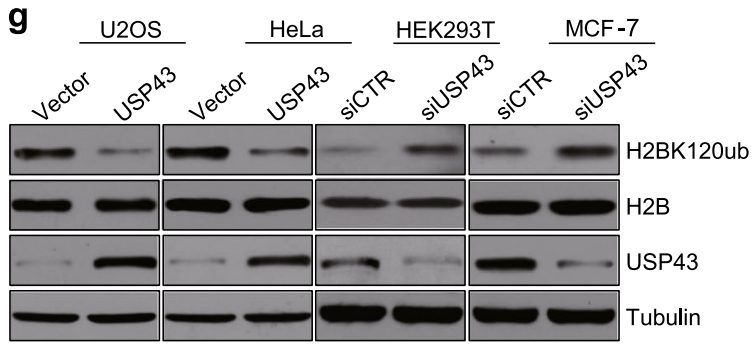

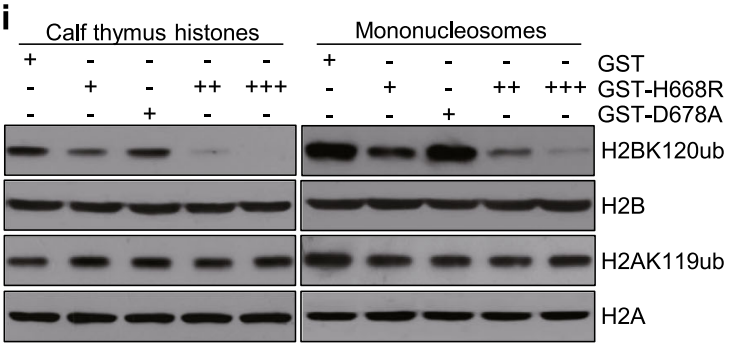

To further strengthen USP43-catalyzed histone deubiquitination, we used site-directed mutagenesis to generate three USP43 mutants: USP43/G36A, USP43/H668R and USP43/D678A. Based on a structural comparison of the putative USP domains of DUBs, ${ }^{16}$ mutation of $\mathrm{H} 668$ or D678 is predicted to interrupt the catalytic core. Western blot analysis of histones extracted from MCF-7 cells revealed that overexpression of wild-type USP43 or USP43/G36A, but not USP43/H668R or USP43/D678A, resulted in a significant decrease in the level of H2BK120ub (Fig. 3h). However, neither overexpression nor knockdown of USP43 was associated with any evident change in H2AK119ub, another important histone ubiquitination (Fig. 3h). Moreover, in vitro deubiquitination experiments carried out by incubating GST-USP43 or GST-USP43/D678A with calf thymus histones or mononucleosomes purified from HeLa cells followed by western blot analysis revealed that recombinant wild-type USP43, but not USP43/D678A, could efficiently remove ubiquitin from $\mathrm{H} 2 \mathrm{BK} 120 \mathrm{ub}$ in a dose-dependent manner (Fig. 3i). Together, these results accord with the observations from SILAC-LC-MS/MS and HSC and support the notion that USP43 
Fig. 3 USP43 is a deubiquitinase acting on H2BK120ub. a The expression of the indicated proteins was measured by western blotting in MCF7 cells following overexpression or knockdown of USP43. b The experimental workflow of SILAC-LC-MS/MS for quantification of histone lysine ubiquitination. c The ratio of the light and heavy peptides identified in SILAC-LC-MS/MS. d Full MS and MS/MS spectra of H2BK120ub in SILACLC-MS/MS. $b$ and $y$ represent the cleavage points of the peptide backbone when the charge is retained at the $\mathrm{N}$ - or $\mathrm{C}$-terminal fragments of the peptide, respectively. e HeLa cells were transfected with siRNA pools against DUBs and immunofluorescently stained with antibodies against H2BK120ub (green). DAPI staining was included to visualize the nucleus (blue). The images represent one field under microscopy in each group (left). Bar, $100 \mu \mathrm{m}$. High-content screening (HCS) with automatic image processing was applied to determine the mean immunofluorescence intensity per cell (right). Error bars represent mean \pm SD for triplicate experiments $\left({ }^{* * *} p<0.001\right)$. f The level of the indicated histone marks or proteins was measured by western blotting in HeLa cells transfected with siRNAs against USP12, USP22, USP43 or USP30. $\mathbf{g}$ The levels of the indicated histone marks or specific proteins was measured by western blotting in U2OS or HeLa cells transfected with FLAG-USP43 expression construct or in HEK293T or MCF-7 cells treated with USP43 siRNA. $\mathbf{h}$ The levels of the indicated histone marks or proteins was measured by western blotting in MCF-7 cells transfected with the indicated expression constructs or specific siRNAs. i In vitro deubiquitination assays with bacterially expressed GST-fused proteins as indicated and calf thymus histones or mononucleosomes. The reaction was analyzed by western blotting with antibodies against the indicated histone marks or proteins

\begin{tabular}{|lll|}
\hline Table 1 & Ubiquitinated lysine sites identified & in SILAC-LC-MS/MS \\
\hline Protein & Position & Modified sequence \\
\hline H2B & 120 & _AVTK(gl)YTSSK_ \\
H3 & 56 & _YQK(gl)STELLIR_ \\
H4 & 31 & _DNIQGITK(gl)PAIR_ \\
H2B & 108 & _PGELAK(gl)HAVSEGT_ \\
H2A & 118 & _GVLPNIQAVLLPK(gl)K_ \\
H2A & 119 & _K(gl)TESHHK_ \\
\hline
\end{tabular}

negatively regulates $\mathrm{H} 2 \mathrm{BK} 120 \mathrm{ub}$ through its deubiquitinase activity.

The USP43/NuRD complex coordinates histone deubiquitination and deacetylation

To provide further support for USP43-catalyzed H2BK120ub deuibiquitination and to explore the functional significance of the physical interaction between USP43 and the NuRD complex, we studied the effects of gain- or loss-of-USP43 function in MCF-7 cells. Western blot analysis of histones extracted from these cells revealed that overexpression of USP43 resulted in a decrease in the level of not only H2BK120ub but also H3 acetylation (H3ac). In contrast, knockdown of USP43 led to an increase in the level of not only H2BK120ub but also H3ac (Fig. 4a), suggesting USP43 is associated with cooperative deubiquitinase and deacetylase activities.

To substantiate these observations, USP43-containing protein complex and the NuRD complex were IP from MCF-7 cells expressing FLAG-USP43 or FLAG-MTA3, respectively, with antiFLAG. The IPs were incubated with calf thymus histones or mononucleosomes derived from HeLa cells, and the enzymatic activities of the immunocomplexes were analyzed by western blotting for H2BK120ub and H3ac. As expected, the USP43containing complex possessed an enzymatic activity that led to a significant decrease in the level of H2BK120ub. Remarkably, the IPs also exhibited dose-dependent deacetylase activity for H3ac (Fig. 4b). Analogously, the MTA3-containing NuRD complex possessed not only deacetylase activity for $\mathrm{H} 3 \mathrm{ac}$ but also deubiquitinase activity for H2BK120ub (Fig. 4b). Moreover, an anti-FLAG antibody was used to precipitate MTA3containing NuRD complex from WT or USP43-KO MCF-7 cells expressing FLAG-MTA3, or to precipitate USP43-containing protein complex from WT or MTA3-KO MCF-7 cells expressing FLAG-USP43. Analysis of the IPs after incubation with calf thymus histones by western blotting with anti-H2BK120 and anti-H3ac showed severely compromised interdependence of such activities (Supplementary information, Figure S4). These experiments demonstrate that the USP43/NuRD complex is essential for crosstalk between histone deubiquitination and deacetylation.

We further performed immunodepletion assays in which the IPS were incubated with anti-USP43- or anti-MTA3-conjugated protein A-Sepharose beads. After two rounds of incubation and the removal of the resins by centrifugation, the supernatants were then used to perform deubiquitinase and deacetylase with histone substrates. The results showed that immunodepletion of USP43 resulted in a drastic decrease in the deacetylation activity of the USP43-containing complex and immunodepletion of MTA3 led to a significant loss in the deubiquitination activity of the MTA3containing protein complex (Fig. 4c). These experiments strongly support the notion that USP43 and the NuRD complex are both physically and functionally associated.

Genome-wide analysis of the transcriptional targets of the USP43/ NuRD complex

To further explore the functional significance of the physical association between USP43 and the NuRD complex, we next analyzed the genome-wide transcriptional targets of USP43. To this end, we first performed chromatin IP (ChIP)-based deep sequencing (ChIP-seq) in MCF-7 cells using antibodies against USP43. Following ChIP, USP43-associated DNAs were amplified using non-biased conditions, labeled, and then sequenced using HiSeq2500. Using MACS Version 2 and with a $p$-value cutoff of $10^{-3}$, we identified 31,556 USP43-specific binding peaks (Fig. 5a). The data for USP43 were then cross-analyzed with our previously published ChIP-seq data for MTA3 in MCF-7 cells (GSE67206) ${ }^{44}$ for overlapping DNA sequences/gene promoters. These promoters were considered to be targets of the USP43/NuRD complex. These experiments identified a total of 1243 target genes, which were classified by gene ontology with DAVID (https://david.ncifcrf.gov/) into different KEGG pathways (Fig. 5b). These KEGG pathways include PI3K-AKT, focal adhesion and cell cycle pathways that are critically involved in cell proliferation, survival, migration and invasion (Fig. 5b). Significantly, analysis of the genomic signatures of USP43 and MTA3 revealed a similar binding motif for these two proteins (Fig. $5 \mathrm{C}$ ), strongly supporting the physical interaction and functional connection between USP43 and MTA3. Quantitative ChIP ( $\mathrm{qChIP}$ ) analysis in MCF-7 cells using specific antibodies against USP43 or MTA3 on selected genes including EGFR, MAD1L1, TNC, NTF3 (Fig. 5d), BUB3, ABL1, COL27A1, PHLPP1, CAMK2B, CALM2, ATG12, FYN and RALA (Supplementary information, Figure $\mathrm{S} 5 \mathrm{a}$ ) showed strong enrichment of USP43 and MTA3 on the promoters of these genes, validating the ChIP-seq results.

To verify that USP43 and MTA3 exist in the same protein complex on target gene promoters, we performed sequential ChIP or ChIP/Re-ChIP on the four representative target genes, EGFR, MAD1L1, TNC and NTF3. In these experiments, soluble chromatin was first IP with antibodies against USP43 and the IPs were subsequently re-IP with antibodies against MTA3. The results 
a
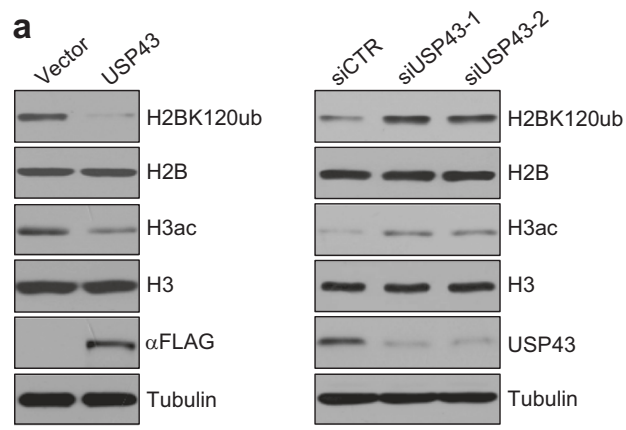

C
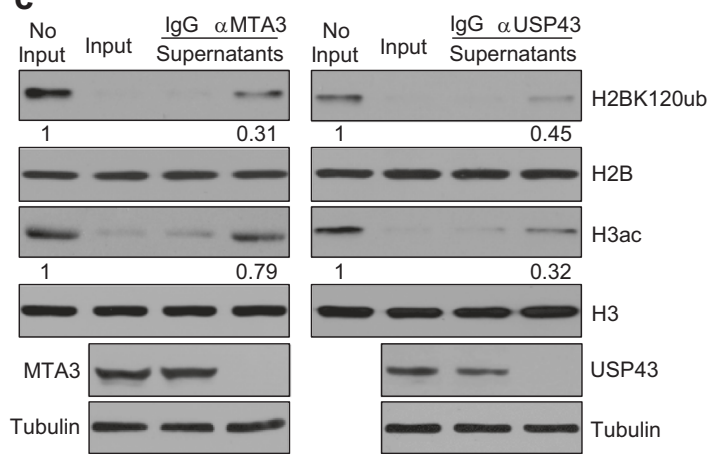

b

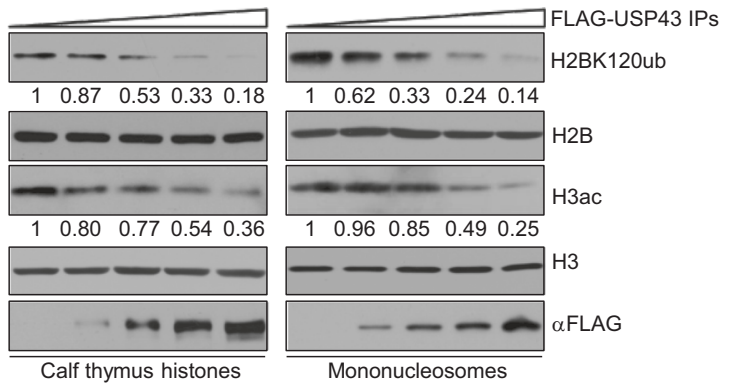

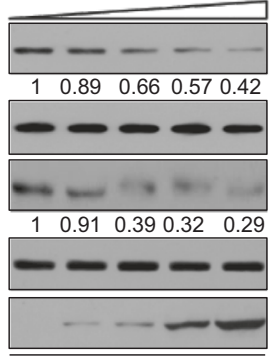

Calf thymus histones

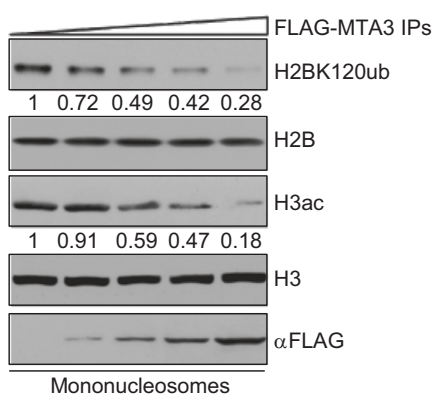

Fig. 4 The USP43/NuRD complex coordinates histone deubiquitination and deacetylation. a MCF-7 cells were transfected with FLAG-USP43 or treated with USP43 siRNAs for the measurement of the indicated histone marks or proteins by western blotting. $\mathbf{b}$ Cellular extracts from MCF7 cells overexpressing FLAG-USP43 (upper) or FLAG-MTA3 (lower) were immunopurified on an anti-FLAG affinity column and eluted with FLAG peptides. The eluates were incubated with calf thymus histones or mononucleosomes isolated from HeLa cells in histone deubiquitination or histone deacetylation assay buffer. The reaction was analyzed by western blotting with antibodies against the indicated histone marks or proteins. The bands were quantified with ImageJ software and the numbers indicate the relative levels of the indicated histone marks. $\mathbf{c}$ Immunodepletion assays in MCF-7 cells with anti-MTA3- or anti-USP43-conjugated protein A-Sepharose beads followed by incubation with calf thymus histones in histone deubiquitination or histone deacetylation assay buffer. The reaction was analyzed by western blotting with antibodies against the indicated histone marks or proteins. The efficiency of the immunodepletion was examined by western blotting of the supernatants

of these experiments indicate that the EGFR, MAD1L1, TNC and NTF3 promoters that were IP with antibodies against USP43 could be re-IP with antibodies against MTA3, whereas anti-USP43 IP target promoters could not be detected in the supernatants with antibodies against MTA3 (Fig. 5e). Similar results were obtained when the initial ChIP was carried out with antibodies against MTA3 (Fig. 5e), supporting the coexistence of USP43 and MTA3 on the promoter of target genes and validating the finding that $E G F R$, MAD1L1, TNC and NTF3 are targeted by the USP43/NuRD complex. Moreover, analysis by real-time reverse transcription (RT)-PCR in MCF-7 cells showed that knockdown of either USP43 or MTA3 was associated with an increased expression of EGFR, MAD1L1, TNC, NTF3 (Fig. 5f), BUB3, ABL1, COL27A1, PHLPP1, CAMK2B, CALM2, $A T G 12, F Y N$ and RALA (Supplementary information, Figure $\mathrm{S} 5 \mathrm{~b}$ ). Consistently, the levels of both H2BK120ub and H3ac were also markedly increased at the four representative target promoters upon knockdown of either USP43 or MTA3 (Fig. 5g). Together, these results support our observations that USP43 and the NuRD complex are physically associated and functionally connected to repress downstream target genes including EGFR, MAD1L1, TNC and NTF3.

USP43 inhibits the proliferation and invasion of breast cancer cells in vitro and suppresses the growth and metastasis of breast cancer in vivo

The identification of EGFR as a target of the USP43/NuRD complex and the well-documented role of EGFR in the development and progression of breast cancer suggested that USP43 might also participate in the regulation of breast cancer growth and metastasis. We therefore first tested the effect of the USP43/ NuRD complex on EGFR protein levels following infection of MDAMB-231 cells with lentiviruses carrying control short hairpin RNA (shRNA)/vector, USP43 shRNA, USP43, and/or MTA3 shRNA, or EGFR. Western blotting showed that the level of EGFR and AKT/ S473 phosphorylation increased upon USP43 depletion. Conversely, USP43 overexpression led to a decrease in the level of EGFR and AKT/S473 phosphorylation, which could be abrogated by simultaneous depletion of MTA3 or overexpression of EGFR (Fig. 6a).

To further explore the functional significance of the USP43/ NuRD complex in breast carcinogenesis, MDA-MB-231 or MCF-7 cells were infected with lentiviruses carrying control shRNA/vector, USP43 shRNA, USP43, and/or EGFR shRNA, MTA3 shRNA or EGFR, and cell proliferation was examined by MTS colorimetric assays. The results showed that USP43 overexpression had a strong inhibitory effect on the proliferation of breast cancer cells, an effect that was probably mediated through the interaction of USP43 with the NuRD complex because knockdown of MTA3 led to a diminished effect. In addition, the growth inhibitory effect of USP43 overexpression appeared, at least in part, to be through its repression of EGFR, as simultaneous overexpression of EGFR could rescue the phenotype. Consistently, USP43 depletion promoted cell proliferation, an effect that could be abrogated, at least partially, by co-knockdown of EGFR (Fig. 6b; Supplementary information, Figure S6a). Colony formation assays also showed that USP43 depletion was associated with an increase in colony 

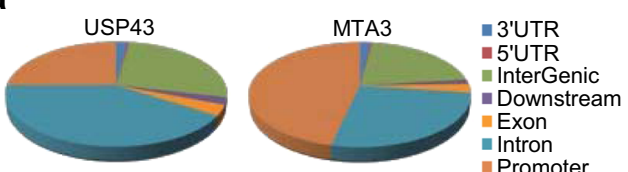

C

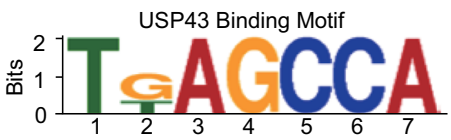

MTA3 Binding Motif

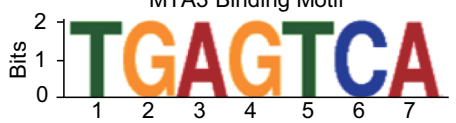

d

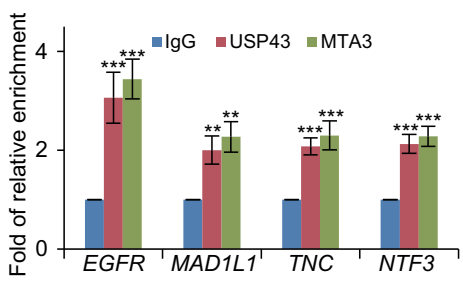

b
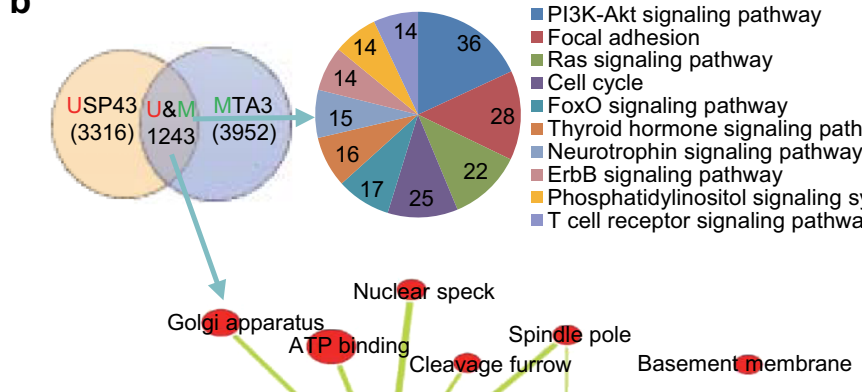

Phosphatidylinositol-3,4-trisphosphate binding Extrinsic component of membrane

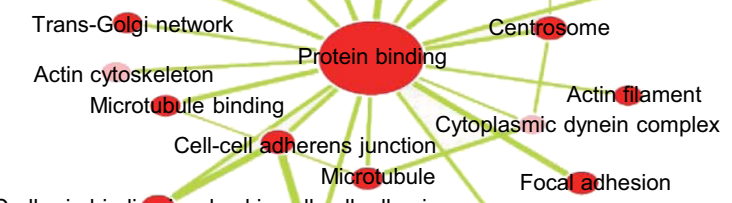

Cadherin binding Involved in cell-cell adhesion Cell-celladhesion
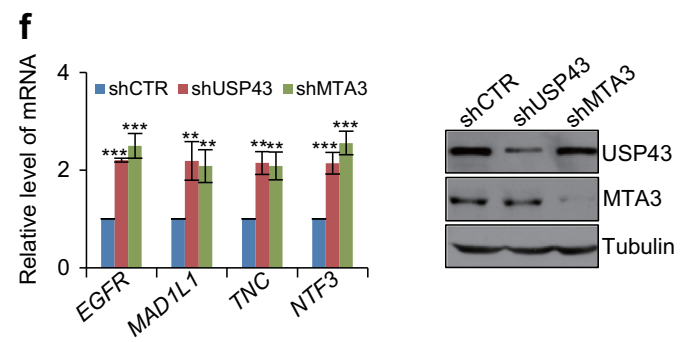

e
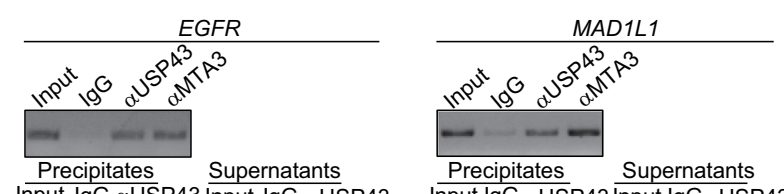
Input IgG $\alpha \mathrm{USP} 43$ Input IgG $\alpha \mathrm{USP} 43$

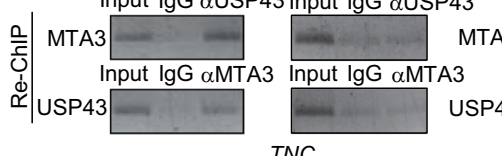

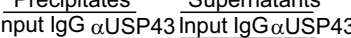
MTA3
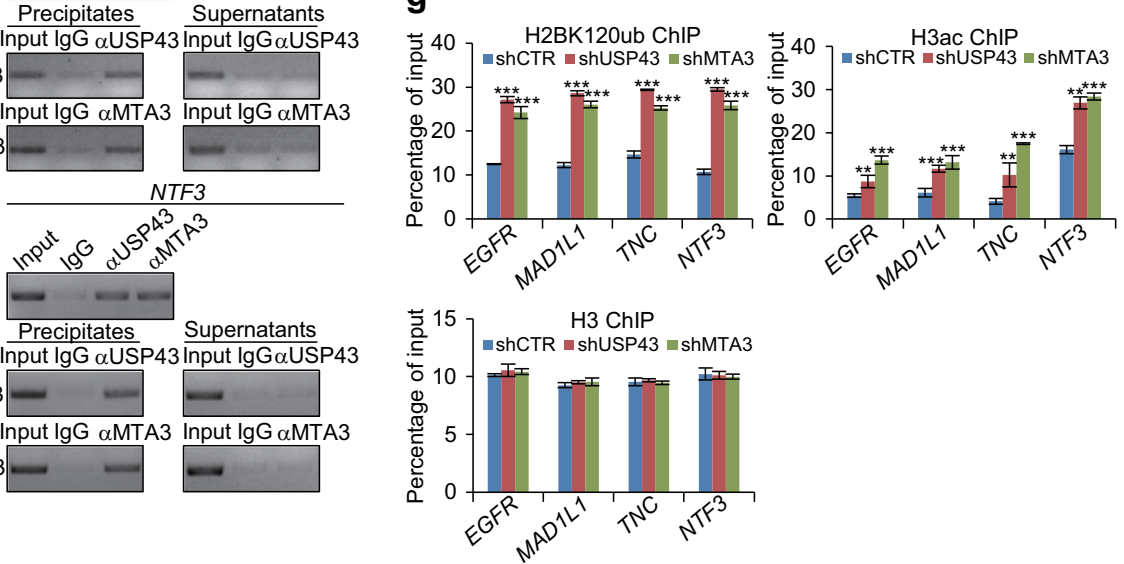

Fig. 5 Genome-wide analysis of the transcriptional targets of the USP43/NuRD complex. a ChIP-seq analysis of the genomic distribution of USP43 and MTA3 in MCF-7 cells. b Venn diagrams of overlapping target genes of USP43 and MTA3 in MCF-7 cells (upper left). The 1243 overlapping target genes of USP43/MTA3 were clustered into KEGG pathways (upper right) or functional groups (lower). The size of the circles represents gene number in each functional group, the depth of the color represents statistical significance, and the thickness of the lines between two functional groups represents the strength of the correlation. c MEME analysis of the DNA-binding motifs of USP43 and MTA3. d Verification of the ChIP-seq results by qChIP analysis of the indicated genes in MCF-7 cells. Results are represented as fold change over control with GAPDH as a negative control. Error bars represent mean \pm SD for triplicate experiments $\left({ }^{* *} p<0.01,{ }^{* * *} p<0.001\right)$. e Soluble chromatin from MCF-7 cells was prepared for ChIP and Re-ChIP assays with antibodies against the indicated proteins. $f$ Real-time RT-PCR measurement of the expression of the indicated genes in MCF-7 cells infected with lentiviruses carrying control, USP43 or MTA3 shRNA. Error bars represent mean \pm SD for triplicate experiments $\left({ }^{* *} p<0.01,{ }^{* * *} p<0.001\right)$. The knockdown efficiency was validated by western blotting. g qChIP analysis on the selected promoters using antibodies against H2BK120ub, H3ac or H3 in MCF-7 cells infected with lentiviruses carrying control shRNA, USP43 shRNA or MTA3 shRNA. H3 was detected as an internal control. Results are represented as fold change over control with GAPDH as a negative control. Error bars represent mean \pm SD for triplicate experiments $\left({ }^{* *} p<0.01\right.$, $\left.{ }^{* * *} p<0.001\right)$ 


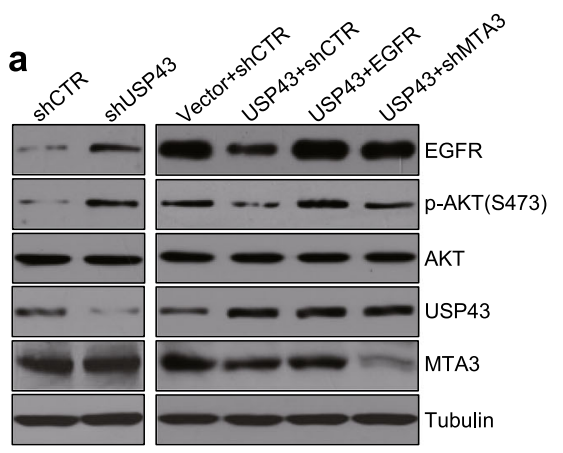

b
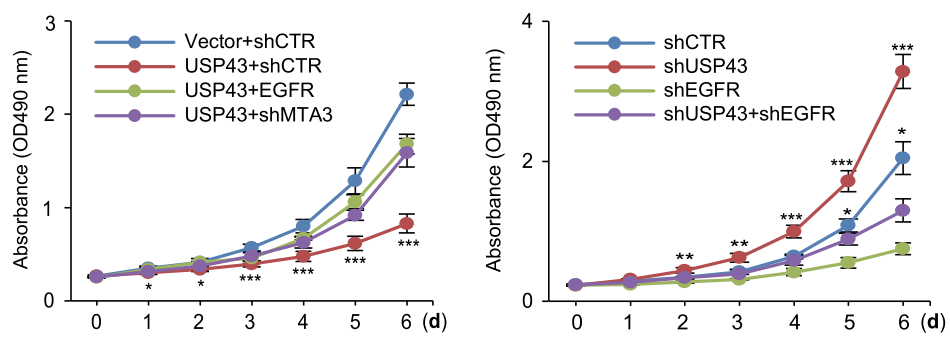

c
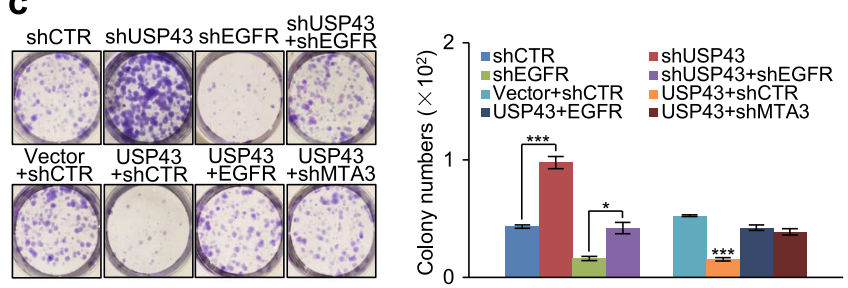

e
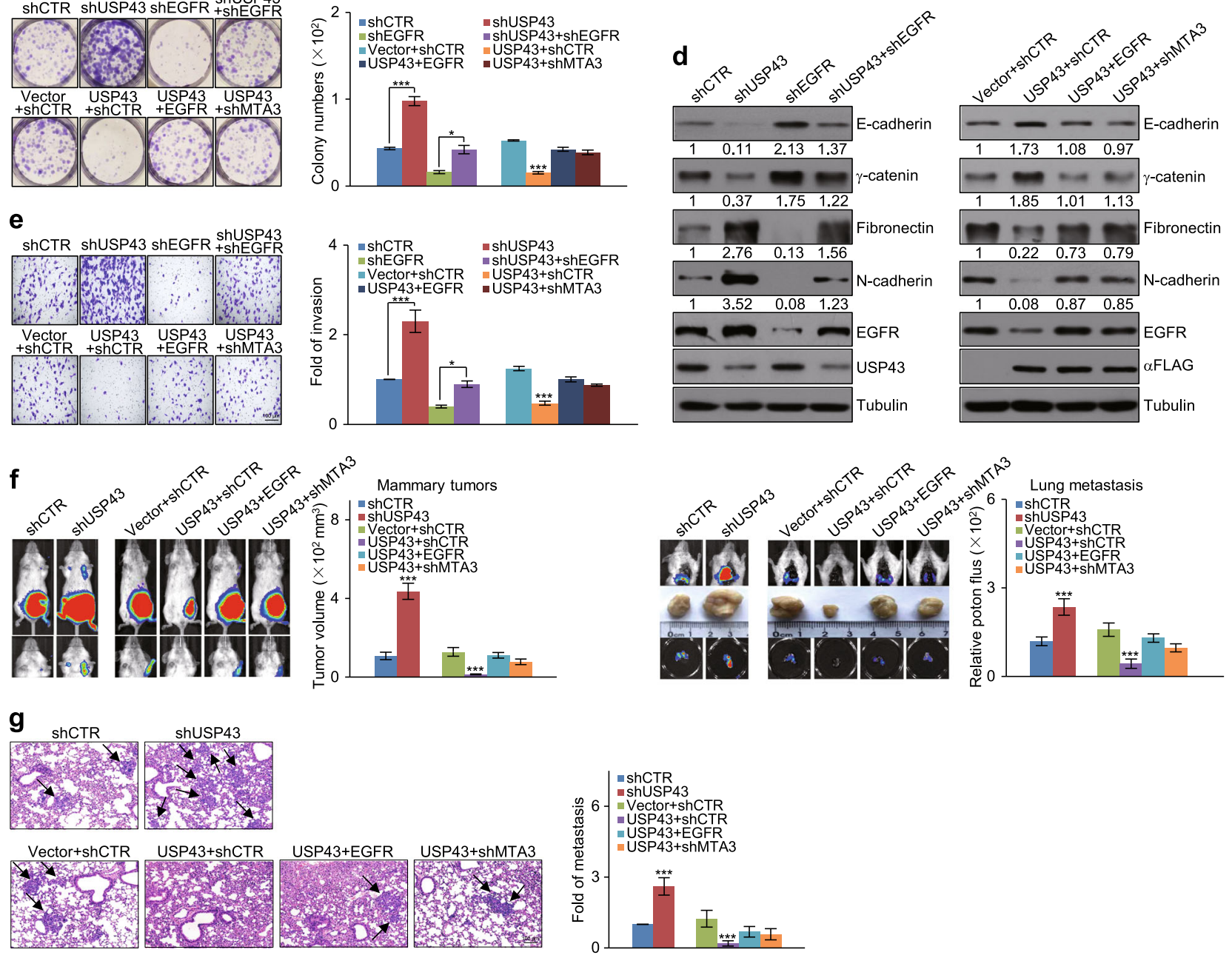

Fig. 6 USP43 inhibits the proliferation and invasion of breast cancer cells in vitro and suppresses the growth and metastasis of breast cancer in vivo. a MDA-MB-231 cells were infected with lentiviruses carrying control shRNA/vector, USP43 shRNA, USP43, and/or EGFR or MTA3 shRNA for western blotting with antibodies as indicated. $\mathbf{b}$ MTS assays for the growth of MDA-MB-231 cells infected with lentiviruses carrying the indicated expression constructs and/or specific shRNAs. Error bars represent the mean \pm SD for three independent experiments $\left({ }^{*} p<0.05,{ }^{* *} p\right.$ $\left.<0.01,{ }^{* * *} p<0.001\right)$. c MDA-MB-231 cells infected with lentiviruses carrying the indicated expression constructs and/or specific shRNAs were cultured for 14 days before staining with crystal violet and counting for colony numbers. Error bars represent the mean \pm SD for three independent experiments (*** $<0.001$ ). d MDA-MB-231 cells were infected with lentiviruses carrying the indicated expression constructs and/ or specific shRNAs for the measurement of the expression of the indicated epithelial/mesenchymal markers by western blotting. The bands were quantified with ImageJ software. The numbers indicate the relative levels of the indicated proteins. e MDA-MB-231 cells were infected with lentiviruses carrying the indicated expression constructs and/or specific shRNA for transwell invasion assays. The invaded cells were stained and counted. The images represent one microscope field in each group. Error bars represent mean \pm SD for triplicate experiments $\left({ }^{* * *} p<0.001\right)$. Bar, $100 \mu \mathrm{m}$. f MDA-MB-231-Luc-D3H2LN cells infected with lentiviruses carrying the indicated expression constructs and/or specific shRNA were inoculated orthotopically onto the abdominal mammary fat pad of 6-week-old female SCID mice. Primary tumor size was measured and metastases were quantified using bioluminescence imaging after 6 weeks of initial implantation. Representative primary tumors and bioluminescent images are shown. Error bars represent mean \pm SD for three independent measurements $(* * * p<0.001)$. g Representative lung metastasis specimens were sectioned and stained with H\&E. Bar, $100 \mu \mathrm{m}$. Error bars represent mean \pm SD $(* * *<<0.001)$ 
number, which was partially attenuated upon co-knockdown of EGFR. In contrast, USP43 overexpression was associated with a decrease in colony number, an effect that could be abrogated by simultaneous depletion of MTA3 or, at least partially, by overexpression of EGFR (Fig. 6c; Supplementary information, Figure S6b). Together, these results support a role for USP43 in the inhibition of cell proliferation and suggest that USP43 does so through its interaction with the NuRD complex and downregulation of target genes including EGFR.

The epithelial-mesenchymal transition (EMT) is a hallmark of cancer and an early event in cancer invasion and metastasis. ${ }^{45}$ To investigate a possible role for USP43 in invasion and metastasis of breast cancer, the expression of epithelial/mesenchymal markers was analyzed by western blotting in MDA-MB-231 or MCF-7 cells. We found that depletion of USP43 resulted in a reduction of epithelial markers including $\mathrm{E}$-cadherin and $\mathrm{Y}$-catenin and an induction of mesenchymal markers including fibronectin and N-cadherin, which were partially attenuated by coknockdown of EGFR (Fig. 6d; Supplementary information, Figure S6c). Conversely, overexpression of USP43 was associated with an induction of the epithelial markers and reduction of the mesenchymal markers. However, simultaneous depletion of MTA3 or overexpression of EGFR counteracted the effect of USP43 overexpression on the expression patterns of the epithelial/ mesenchymal markers (Fig. 6d; Supplementary information, Figure S6c). These results support a role for USP43 in the inhibition of the EMT, indicating that USP43 does so, through its interaction with the NuRD complex and downregulation of target genes including EGFR.

We then investigated the role of USP43 in the cellular behavior of breast cancer cells in vitro using transwell invasion assays. We found that USP43 knockdown was associated with an increase in the invasive potential of MDA-MB-231 cells, whereas USP43 overexpression was accompanied by a decrease in the invasive potential of such cells (Fig. 6e). Moreover, in agreement with the existence of a functional link between USP43 and EGFR, the increase in invasive potential associated with USP43 knockdown could be rescued, at least partially, by co-knockdown of EGFR and the inhibitory effect of USP43 overexpression on the invasive potential of MDA-MB-231 cells was partially offset by EGFR overexpression. The inhibitory effect of USP43 overexpression on invasiveness appeared to be achieved through concerted action with the NuRD complex because the effect diminished when MTA3 was concomitantly knocked down in MDA-MB-231 cells (Fig. 6e). Taken together, these results support a role for USP43 in regulating the invasive potential of breast cancer cells and suggest that it does so through its association with the NuRD complex and downregulation of target genes including EGFR.

To investigate the role of USP43 in breast cancer metastasis in vivo, MDA-MB-231 cells that had been engineered to stably express firefly luciferase (MDA-MB-231-Luc-D3H2LN, Xenogen Corporation) were infected with a control lentivirus shRNA vector and lentiviruses carrying USP43 shRNA, USP43, and/or MTA3 shRNA or EGFR. These cells were then implanted onto the left abdominal mammary fat pad of 6-week-old female SCID mice $(n=6)$. The growth/dissemination of tumors was monitored weekly by bioluminescence imaging with the IVIS imaging system (Xenogen). Tumor metastasis was defined as any detectable luciferase signal above background and away from the primary tumor site and was measured by quantitative bioluminescence imaging after 6 weeks. We found that USP43 depletion promoted the growth of the primary tumor (Fig. 6f) and lung metastasis (Fig. $6 \mathrm{~g}$ ) of the MDA-MB-231-Luc-D3H2LN tumors. Conversely, USP43 overexpression resulted in growth suppression of the primary tumor (Fig. 6f), as well as lung metastasis (Fig. 6g) of MDAMB-231-Luc-D3H2LN tumors. Remarkably, simultaneous depletion of MTA3 or overexpression of EGFR counteracted the USP43 overexpression-associated suppression of the growth of primary tumors (Fig. 6f) and lung metastases (Fig. 6g). Collectively, these experiments indicate that USP43 suppresses the growth and metastasis of breast cancer and that it does so through its interaction with the NuRD complex and repression of target genes including EGFR.

Cancer-associated mutations of USP43 affect its subcellular localization and/or epigenetic regulatory function

To extend our observations to pathologically relevant contexts, we next investigated the genetic alterations of USP43 in various human carcinoma samples from the clinic. Analysis of the cBioPortal for Cancer Genomics (http://www.cbioportal.org/) for USP43 mutations revealed that mutation in the $\mathrm{N}$-terminal of USP43 is relatively rare in various human carcinoma samples, and that mutations were observed at high frequency in amino-acid residues located more C-terminally including Q276R, F402L, Q533H, H660N, D678N, V833G, G889R and V1023R (Fig. 7a). Thus, we generated USP43 mutant for each of these eight mutations and first examined their subcellular localizations. Transfection of FLAG-tagged wild-type USP43 and USP43 mutants into MCF-7 cells and analysis by immunofluorescent staining with anti-FLAG showed that wild-type USP43, USP43/H660N and USP43/D678N were present in both the cytoplasm and nucleus, the remaining USP43 mutants were mainly localized in the cytoplasm (Fig. 7b). Western blot analysis of the cytoplasmic and nuclear extracts of these cells showed consistent results (Fig. 7c). We then tested the interaction between the eight USP43 mutants and the $14-3-3 \beta / \varepsilon$ heterodimer or MTA3. IPs in MCF-7 cells expressing the FLAGtagged USP43 mutants with anti-FLAG followed by IB with antibodies against phospho-(Ser/Thr), $14-3-3 \beta, 14-3-3 \varepsilon$ or MTA3 demonstrated that each these mutants, except for USP43/H660N and USP43/D678N, exhibited a higher phosphorylation level, stronger interaction with $14-3-3 \beta / \varepsilon$ heterodimer and weaker association with MTA3, compared with wild-type USP43 (Fig. 7d). Consistently, transfections of FLAG-tagged USP43 mutants into MCF-7 cells and subsequent western blot analysis revealed that almost all of the eight USP43 mutants showed compromised ability to deubiquitinate $\mathrm{H} 2 \mathrm{BK} 120 \mathrm{ub}$ and repress EGFR expression, compared with wild-type USP43, even though USP43/H660N and USP43/D678N, which were found to be enriched in the nucleus, were associated with deacetylase activity for $\mathrm{H3ac}$ (Fig. 7e). Notably, both $\mathrm{H} 660$ and D678 reside in the conserved USP domain of USP43 and are important for the enzymatic activity of DUBs. ${ }^{16}$ Together, these results indicate that cancer-associated USP43 mutants display either aberrant cytoplasmic retention or an impaired deubiquitinase activity toward H2BK120ub. This leads to impaired transcription repression of downstream target genes including $E G F R$, and eventually, contributing to breast carcinogenesis. In agreement, MTS assays showed that all the USP43 mutants largely lost inhibitory effect on MCF-7 cell proliferation (Fig. 7f) and transwell invasion assays revealed that these mutants showed significantly compromised inhibition of the invasive potential of MDA-MB-231 cells (Fig. 7g).

Nuclear USP43 is reduced in breast carcinomas, corresponding to the high expression of EGFR and hyperactivation of AKT

To further extend our observations to pathologically relevant settings, we examined the protein level of USP43 by immunohistochemical staining of human tissue arrays containing a series of carcinoma samples from thyroid, esophagus, stomach, colon, rectum, liver, pancreas, lung, breast and kidney cancer patients. These arrays held at least six samples of each type of carcinoma paired with the adjacent normal tissues. Strikingly, we found that USP43 protein was predominantly present in the cytoplasm of cancer cells, especially in esophagus, colon, rectum, lung, and breast carcinomas, whereas in adjacent cells, abundant USP43 was detected in the nucleus (Fig. 8a). Evidently, the ratio of nuclear/ cytoplasmic USP43 was significantly reduced in esophagus, colon, 
a

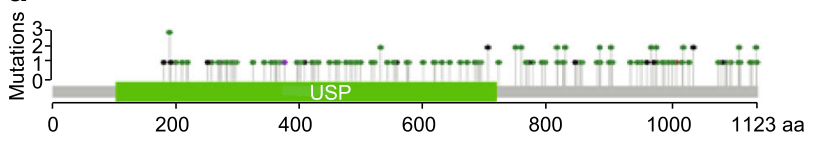

b
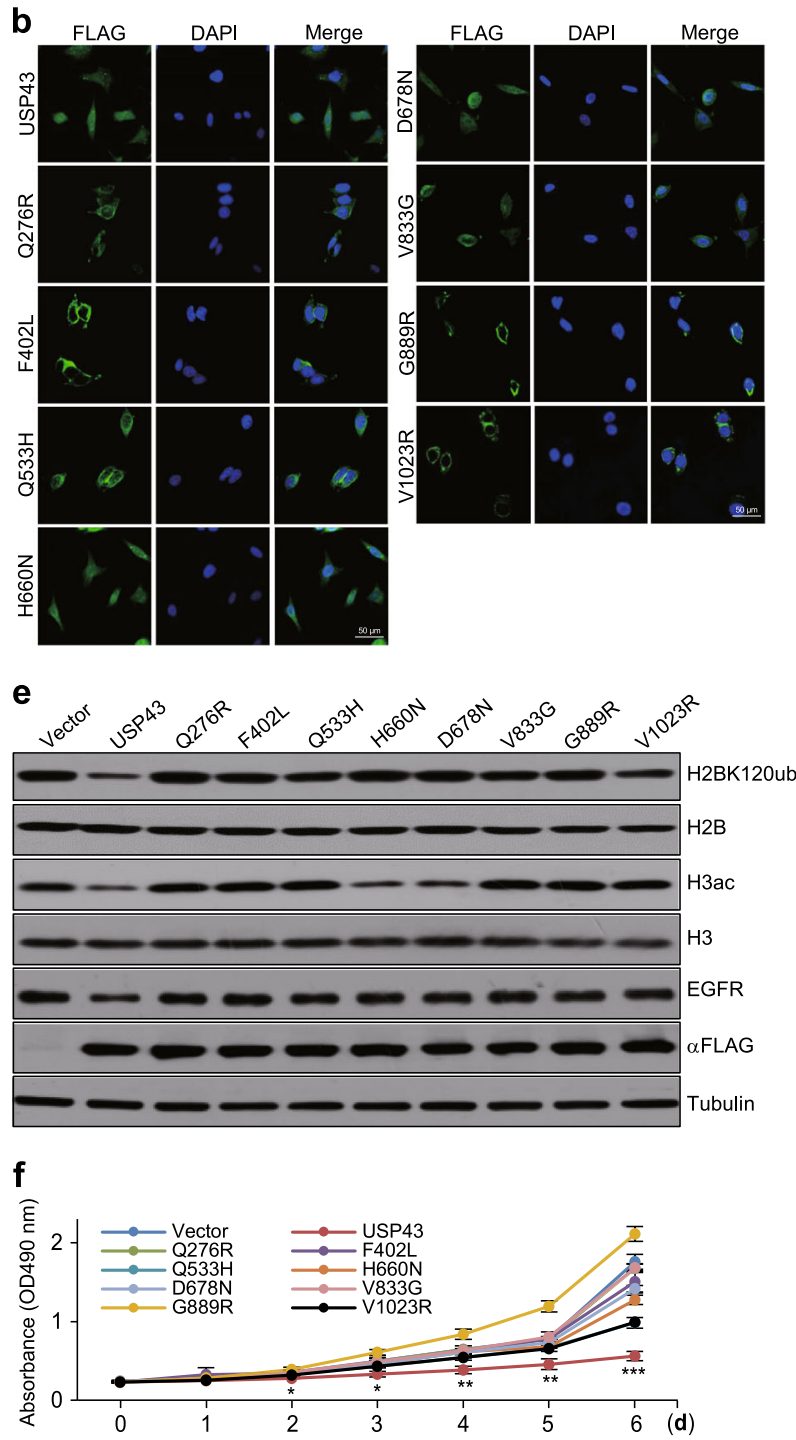

C

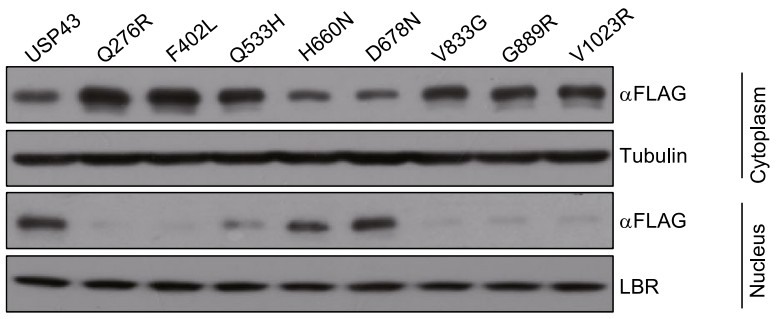

d

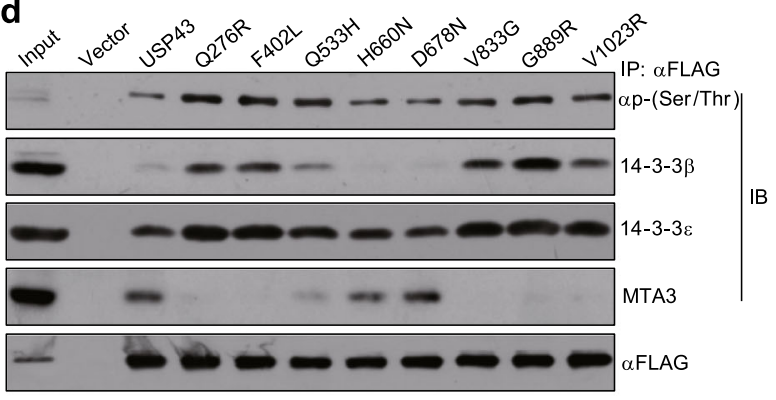

g
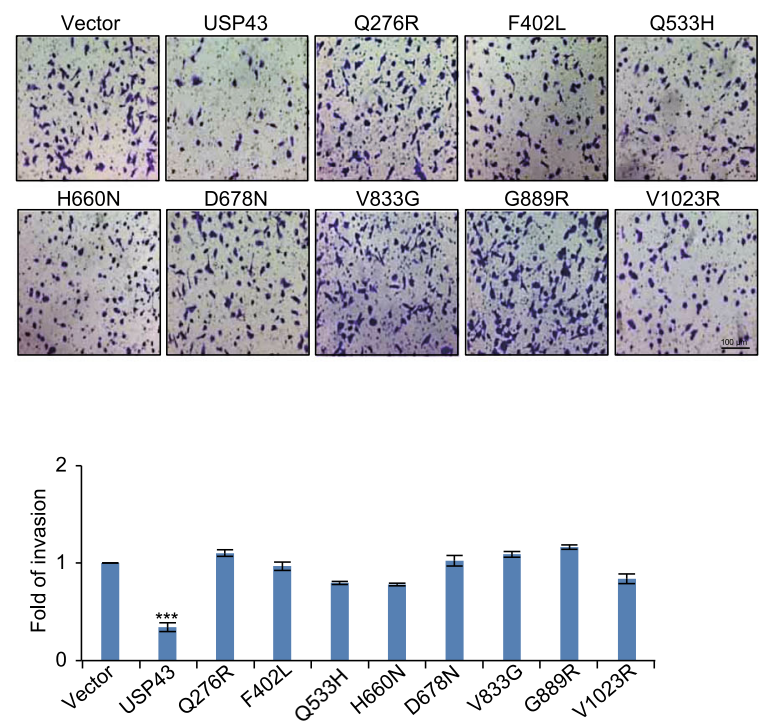

Fig. 7 Cancer-associated mutations of USP43 affect its subcellular localization and/or epigenetic regulatory function. a Analysis of public datasets for spontaneous mutations of USP43 in various types of cancers. b MCF-7 cells were treated with specific siRNA targeting USP43 3'UTR prior to transfection with the indicated FLAG-tagged expression constructs for immunofluorescent staining with anti-FLAG to visualize USP43 (green). DAPI staining was included to visualize the nucleus (blue). c MCF-7 cells were treated with specific siRNA targeting USP43 3'UTR prior to transfection with the indicated FLAG-tagged USP43 expression constructs for western blot analysis of USP43 expression with anti-FLAG antibody in the nucleus and cytoplasm. d MCF-7 cells were treated with specific siRNA targeting USP43 3'-UTR prior to transfection with the indicated FLAG-tagged USP43 expression constructs for co-immunoprecipitation assays with anti-FLAG followed by immunoblotting with antibodies as indicated. e MCF-7 cells were treated with specific siRNA targeting USP43 3'-UTR prior to transfection with the indicated FLAG-tagged expression constructs for the measurement of the level of the indicated histone marks or proteins by western blotting. $\mathbf{f}$ MCF-7 cells were treated with specific siRNA targeting USP43 3'-UTR prior to transfection with the indicated expression constructs for the measurement of the growth curves with MTS assays. Error bars represent mean \pm SD for triplicate experiments $\left({ }^{*} p<0.05,{ }^{* *} p<0.01,{ }^{* * *} p<\right.$ 0.001). g MDA-MB-231 cells were treated with specific siRNA targeting USP43 3'-UTR prior to transfection with the indicated expression constructs for transwell invasion assays. The invaded cells were stained and counted. The images represent one field under microscopy in each group. Error bars represent mean \pm SD for triplicate experiments $\left({ }^{* * *} p<0.001\right)$. Bar, $100 \mu \mathrm{m}$

rectum, pancreas, lung, breast and kidney carcinomas, compared with adjacent normal cells (Fig. 8a).

We then performed immunohistochemical staining of human tissue arrays containing 28 breast carcinoma samples (grade II: 17, grade III: 11) paired with normal mammary tissues, as well as of human tissue arrays containing 137 breast carcinoma samples (grade II: 88, grade III: 49) from breast cancer patients for whom complete medical history was available. We found that nuclear USP43 and the ratio of nuclear/cytoplasmic USP43 were significantly lower in the majority of the 28 breast carcinoma samples 

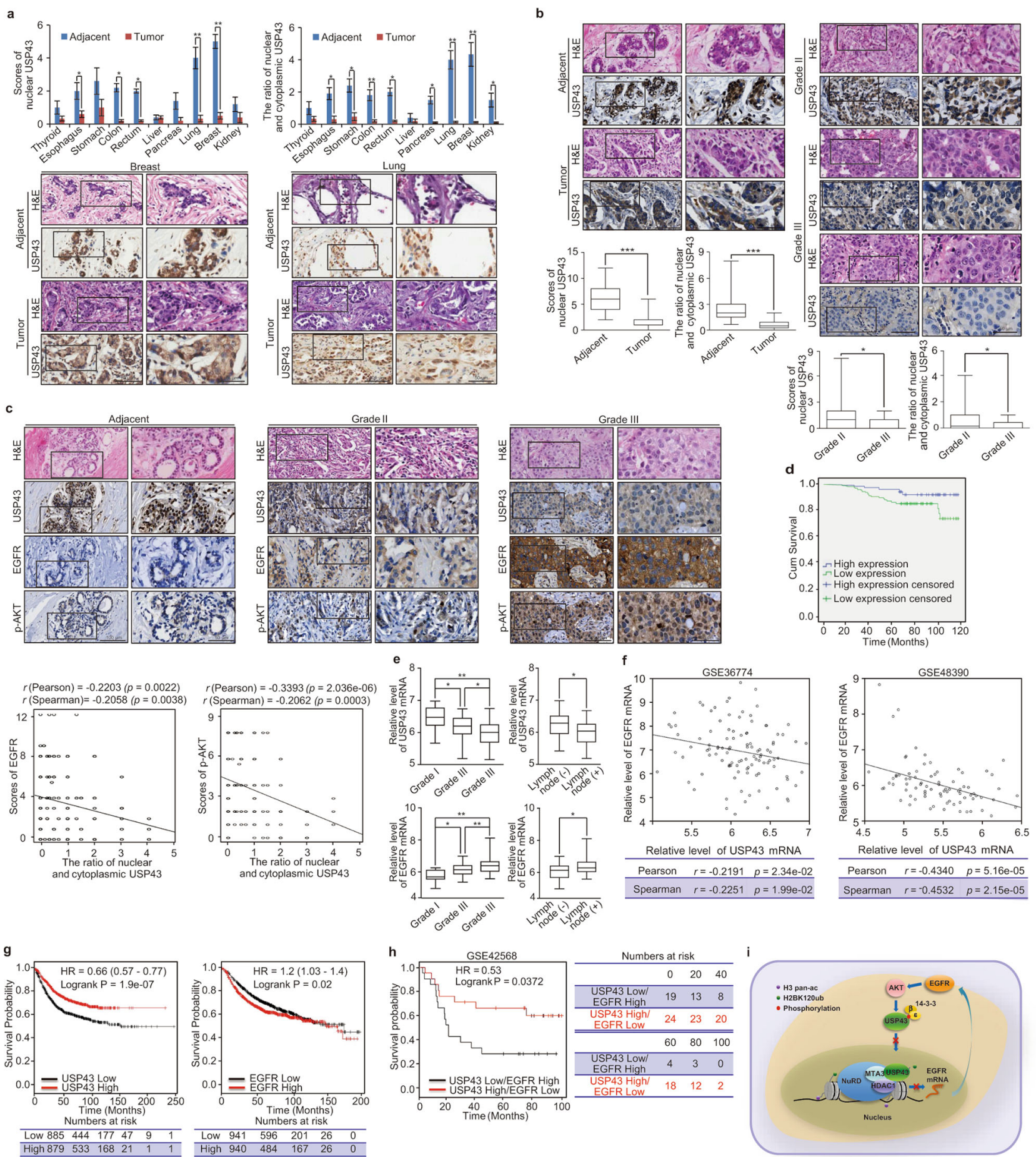
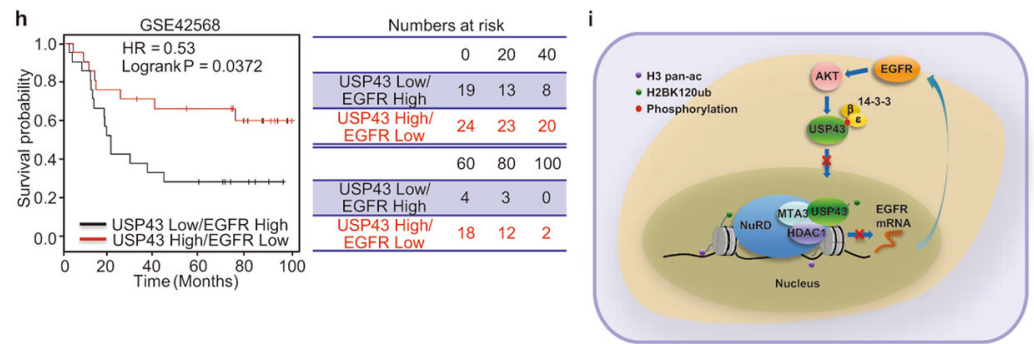

(22/28) compared with adjacent normal tissues. Remarkably, 3 out of the 137 breast carcinomas showed a lack of USP43 staining (Fig. 8b). Significantly, the level of nuclear USP43 negatively correlated with the histological grade of the 165 breast carcinoma samples $(28+137)$ (Fig. 8b). Moreover, the level of nuclear USP43 in these 165 breast carcinoma samples also negatively correlated with tumor size, depth of invasion, lymph node metastasis and advanced TNM stage (Table 2). Consistent with our observations that EGFR is a downstream target of the USP43/NuRD complex and that AKT activation is associated with retention of USP43 in cytoplasm, the ratio of nuclear/cytoplasmic USP43 also negatively correlated with the levels of EGFR expression and AKT phosphorylation in the 165 breast carcinoma samples (Fig. 8c). Furthermore, the overall 5-year survival rate of patients with high nuclear expression of USP43 was significantly higher than patients with low nuclear expression of USP43 (Fig. 8d). Consistently, interrogation of public dataset (GSE36774) found that the level of USP43 shows a negative correlation and EGFR, a positive correlation with the histological grades of breast cancer (Fig. 8e). Strikingly, we found that low USP43 and high EGFR in breast carcinomas strongly correlates with lymph node positivity of the patients (Fig. 8e). 
Fig. 8 Nuclear USP43 is reduced in breast carcinomas and the ratio of nuclear/cytoplasmic USP43 is negatively correlated with high expression of EGFR and hyperactivation of AKT. a Immunohistochemical staining of USP43 in tissue arrays containing various types of carcinomas samples paired with adjacent normal tissues. Each type of carcinoma included at least six paired samples and the scores were determined by evaluating the extent and intensity of immunopositivity and were analyzed by paired samples $t$-test $\left({ }^{*} p<0.05,{ }^{* *} p<0.01\right)$. Samples of tumors and normal tissues from breast and lung were sectioned and stained with H\&E or immunostained with anti-USP43, and representative images are shown. Bar, $100 \mu \mathrm{m}$ (left), $50 \mu \mathrm{m}$ (right). b Immunohistochemical staining of USP43 in tissue arrays containing 28 breast carcinoma samples (grade II: 17, grade III: 11) paired with normal mammary tissues as well as tissue arrays containing 137 breast carcinoma samples (grade II: 88, grade III: 49). Representative images from H\&E staining and immunostaining with anti-USP43 are presented. Bar, $100 \mu \mathrm{m}$ (left); $50 \mu \mathrm{m}$ (right). The scores were determined by evaluating the extent and intensity of immunopositivity and were analyzed by two-tailed paired $t$-test (lower left) or two-tailed unpaired $t$-test (lower right) $\left.{ }^{*} p<0.05,{ }^{* * *} p<0.001\right)$. c Samples from adjacent normal tissues and grade II/III breast cancers were H\&E stained or immunostained with antibodies against USP43, EGFR or p-AKT. Representative images are shown. Bar, $100 \mu \mathrm{m}$ (left); $50 \mu \mathrm{m}$ (right). The ratio of nuclear and cytoplasmic USP43 was plotted against the scores of EGFR or p-AKT. The correlation coefficients were calculated by $\mathrm{R}$ programming. $\mathbf{d}$ Time-to-event data were plotted using Kaplan-Meier curves, and the 5-year survival rate of different groups was compared using the Mantel-Cox log-rank test $\left({ }^{* * *} p<0.001\right)$. The $y$ axis represents the percentage of patients, and the $x$ axis represents the survival in months. e Analysis of public dataset (GSE36774) for the correlation between the level of USP43 or EGFR and the histological grades of breast carcinomas or lymph node metastasis of breast cancer patients $\left.{ }^{*} p<0.05,{ }^{* *} p<0.01\right)$. $\mathbf{f}$ Analysis of public datasets (GSE36774 and GSE48390) for the expression of EGFR and USP43. The relative mRNA level of EGFR was plotted against that of USP43. g Kaplan-Meier survival analysis (http://kmplot.com/analysis/) for the relationship between survival time of breast cancer patients and the expression levels of USP43 or EGFR. h Kaplan-Meier survival analysis of public dataset (GSE42568) for the relationship between survival time and the expression levels of USP43/EGFR in breast cancer. $\mathbf{i}$ The proposed model of the reciprocally inhibitory loop between EGFR/PI3K/AKT and USP43 in breast cancer carcinogenesis

To address the clinicopathological relevance of USP43 and EGFR, we next analyzed public datasets for mRNA levels of USP43 and EGFR in breast carcinomas. Analysis of two published clinical datasets (GSE36774 and GSE48390) revealed a statistically significant negative correlation of expression between USP43 and EGFR in breast carcinomas (Fig. 8f). Strikingly, analysis of public dataset (GSE48390) for USP43 or EGFR expression revealed that expression of USP43 is higher in Luminal A and Luminal B subtypes and lower in HER2-enriched and basal-like subtypes, where the expression of EGFR is high (Supplementary information, Figure S7a). Kaplan-Meier survival analysis (http://kmplot.com/analysis/) demonstrated that either higher USP43 expression (hazard ratio, $\mathrm{HR}=0.66, p=1.9 \mathrm{e}-07$ ) or lower EGFR expression ( $\mathrm{HR}=1.2, p=0.02)$ was associated with a better overall survival for patients with breast cancer (Fig. 8g). Moreover, higher USP43 expression is associated with better relapse-free survival of breast cancer patients of Luminal $A$, Luminal $B$ and even basal-like subtypes (Supplementary information, Figure S7b). Further stratification of patient groups (GSE42568) based on the inverse expression of USP43 and EGFR improved the predictive capability of USP43 ( $\mathrm{HR}=0.53, p=0.0372)$ (Fig. 8h). Collectively, these data are consistent with a role for USP43 in suppressing breast cancer development and progression by targeting the downstream genes including EGFR.

\section{DISCUSSION}

Genetic mutation/deletion of chromosome 17p13.1 is considered as a prototype of genetic abnormalities associated with malignant transformation owing to its recurrent or invariable inclusion of TP53, an extensively studied tumor-suppressor gene that is believed to be the major driver of $17 \mathrm{p} 13.1$ loss-of-functionassociated pathologies. ${ }^{46-48}$ Interestingly, the gene encoding the USP family protein USP43 also maps to this cytological region, suggesting that $17 \mathrm{p} 13.1$ mutations, especially deletions, could entail collateral damage to USP43. Indeed, analysis of public datasets showed that USP43 suffers frequent mutation and/or deletion in various types of tumor, especially in invasive breast carcinoma. Notably, the chromosome region 17 p13.1 encompasses $\sim 4.3$ million base pairs and harbors at least 144 genes including, in addition to TP53 and USP43, important genes such as MED31 and CHD3 (encoding for Mi-2a). It will be a daunting task to determine the scope and variety of the other genes whose mutations/deletions accompany the genetic abnormalities of TP53 in specific tumor types. Nevertheless, it is a safe assumption that, in addition to mutation/deletion of TP53, loss-of-function of one or more of these 144 genes might, at least, also contribute to tumorigenesis. Our finding that USP43 is frequently mutated in a variety of human cancers suggests that USP43 might be another underappreciated candidate tumorsuppressor gene. Moreover, although USP43 is a predicted member of the USP deubiquitinase family, its biological function has remained uncharacterized.

We have found that USP43 is physically associated with the NuRD complex and biochemically catalyzes H2BK120 deubiquitination. We have shown that USP43 and the NuRD complex act in coordination to remove histone ubiquitination and acetylation. The USP43/NuRD complex represses a panel of genes including EGFR that are critically involved in cell proliferation and tumor growth and we have demonstrated that the USP43/NuRD complex inhibits the proliferation and invasion of breast cancer cells in vitro and suppresses the growth and metastasis of breast cancer in vivo. These results support the notion that USP43 is a potent suppressor of breast carcinogenesis and thus USP43 represents another potential tumor suppressor.

Extensive studies by us and others have implicated the NuRD complex in the development and progression of breast cancer. $^{44,49-54}$ The NuRD complex contains several subunits whose pattern of expression is heterogeneous in various cell and tissue types, leading to the proposal that subunit heterogeneity confers these complexes with additional regulatory capacity and with unique functional properties. ${ }^{41,49,55}$ Consistent with this notion, we reported previously that histone demethylases LSD ${ }^{49}$ and JARID $1 B^{50}$ are associated with the NuRD complex, thus expanding its enzymatic repertoire to include, in addition to ATPase and deacetylase, the demethylase activity. Our current inclusion of USP43 in the NuRD complex extends the enzymatic arsenal of the NuRD complex further to include a deubiquitinase activity. It is becoming increasingly clear that the NuRD complex acts as an epigenetic regulatory hub that coordinates different histone modifications and directs distinct "histone crosstalk". However, our ChIP-seq results clearly show an incomplete overlap between USP43 and MTA3 targets, suggesting that NuRD complex could recruit some other transcriptional cofactors, whereas USP43 also could deubiquitinate non-histone proteins independent of each other in some specific physiological states. Further investigations will determine whether the NuRD complex contains further enzymatic activities and additional chromatin modifying capabilities.

Although H2BK120 ubiquitination is catalyzed mainly by the RNF20/40 complex, ${ }^{30}$ at least six USPs, including USP3, USP12, 


\begin{tabular}{|c|c|c|c|c|}
\hline \multirow[t]{2}{*}{ Variables } & \multirow[t]{2}{*}{$n$} & \multicolumn{2}{|c|}{ Nuclear USP43 level } & \multirow[t]{2}{*}{$p$-Value } \\
\hline & & Positive $(n, \%)$ & Negative $(n, \%)$ & \\
\hline \multicolumn{5}{|c|}{ Histological grade } \\
\hline II & 101 & 52 (74.29\%) & 49 (57.65\%) & \multirow[t]{2}{*}{$p=0.023$} \\
\hline III & 54 & $18(25.71 \%)$ & $36(42.35 \%)$ & \\
\hline \multicolumn{5}{|l|}{ Age (years) } \\
\hline$\geq 55$ & 81 & $34(46.58 \%)$ & 47 (57.32\%) & \multirow[t]{2}{*}{$p=0.181$} \\
\hline$<55$ & 74 & 39 (53.42\%) & 35 (42.68\%) & \\
\hline \multicolumn{5}{|l|}{ Location } \\
\hline Left & 87 & 41 (54.67\%) & 46 (58.23\%) & \multirow[t]{2}{*}{$p=0.745$} \\
\hline Right & 67 & $34(45.33 \%)$ & $33(41.77 \%)$ & \\
\hline \multicolumn{5}{|c|}{ Tumor size $(\mathrm{cm})$} \\
\hline $\mathrm{D} \leq 3.3$ & 94 & 57 (79.17\%) & $37(44.58 \%)$ & \multirow[t]{2}{*}{$p<0.001$} \\
\hline $\mathrm{D}>3.3$ & 61 & 15 (20.83\%) & 46 (55.42\%) & \\
\hline \multicolumn{5}{|c|}{ Depth of invasion } \\
\hline T0 and T1 & 40 & $28(39.44 \%)$ & $12(14.63 \%)$ & \multirow[t]{3}{*}{$p<0.001$} \\
\hline $\mathrm{T} 2$ & 103 & 42 (59.15\%) & 61 (74.39\%) & \\
\hline T3 & 10 & $1(1.41 \%)$ & 9 (10.98\%) & \\
\hline \multicolumn{5}{|l|}{ Lymph node } \\
\hline No & 78 & 45 (60.81\%) & 33 (40.74\%) & \multirow[t]{3}{*}{$p=0.044$} \\
\hline N1 & 43 & $16(21.62 \%)$ & 27 (33.33\%) & \\
\hline $\mathrm{N} 2$ and $\mathrm{N} 3$ & 34 & $13(17.57 \%)$ & $21(25.93 \%)$ & \\
\hline \multicolumn{5}{|l|}{ TNM stage } \\
\hline I & 26 & 21 (29.58\%) & $5(6.17 \%)$ & \multirow[t]{3}{*}{$p<0.001$} \\
\hline II & 87 & 37 (52.11\%) & $50(61.73 \%)$ & \\
\hline III & 39 & 13 (18.31\%) & 26 (32.10\%) & \\
\hline
\end{tabular}

USP22, USP44, USP46 and USP49, have been reported to be able to deubiquitinate $\mathrm{H} 2 \mathrm{BK} 120$. Our identification of USP43 as another eraser for H2BK120ub adds to the complexity of the regulation of this histone mark. The biological significance of the multifarious requirement for the regulation of $\mathrm{H} 2 \mathrm{BK} 120 \mathrm{ub}$ deubiquitination is currently unknown. It is possible that different USPs act on H2BK120ub in a tissue/gene-specific manner and/or in responding to a different extracellular stimulus/cellular environment. Our study indicates that the function of USP43 is tightly linked to the EGFR/PI3K/AKT signaling pathway in which USP43 is phosphorylated by AKT, resulting in its binding to $14-3-3 \beta / \varepsilon$ heterodimer and sequestration in cytoplasm. Logically, dephosphorylation of USP43 leads to its nuclear translocation and interaction with the NuRD complex. Due to the scope of our current investigation, we have not identified the phosphatase involved in the regulation of USP43's nuclear translocation. Future investigations of this issue will surely provide a more detailed account of the regulation of the cellular function of this deubiquitinase. Moreover, our experiments showed that elevated USP43 could also affect the ubiquitination level of H3K56ub and H4K31ub. Although, due to the lack of the appropriate antibodies, we were unable to study the regulation of these histone marks by USP43 and further study of this is warranted.

Mutation/amplification of EGFR is also a frequent event in malignancies from a broad spectrum of tissue origins and this aberration is often correlated with advanced stages of cancer progression and poor prognosis. ${ }^{1-3,9,56}$ Constitutive activation of EGFR leads to hyperactivation of downstream signaling pathways including the PI3K-AKT pathway that are critically involved in cell proliferation and malignant transformation. ${ }^{4-6}$ Remarkably, gain-offunction mutation/amplification of $P I 3 K_{,}^{10,11,57}$ the positive regulator of $\mathrm{AKT}^{7}$ and loss-of-function mutation of $\mathrm{PTEN}^{12}$ a negative regulator of $\mathrm{AKT}^{8}$ also frequently occur in human cancers, all of which could lead to hyperactivation of AKT. However, these genetic alterations cannot fully account for the hyperactivation of the EGFR/ PI3K/AKT cascade. ${ }^{13}$ According to our model, hyperactivation of AKT would result in hyperphosphorylation and cytoplasmic retention of USP43, leading to lost transcriptional regulation by USP43. On the other hand, loss-of-function mutation/deletion of USP43 will render lost transcriptional repression of genes including EGFR and diminished negative regulation of the EGFR/PI3K/AKT signaling by USP43. If our interpretation is correct, there appears to exist a reciprocally inhibitory loop inside the cell in which the activation of EGFR/PI3K/AKT signaling sequestrates USP43 in the cytoplasm thus inhibiting the transcription regulatory function of USP43, leading to cell proliferation and invasion. On the other hand, USP43 translocates into the nucleus and transcriptionally represses EGFR, leading to the inhibition of cell proliferation and invasion. According to this model, the imbalance of this loop, either due to hyperactivation of EGFR/PI3K/AKT signaling resulting from gain-offunction mutations or amplification of these components, or to diminished transcriptional regulatory function of USP43 resulting from loss-of-function mutations or deletion, strongly favors breast carcinogenesis. These scenarios place USP43 into a critical node in the hierarchical regulatory network of the EGFR/PI3K/AKT signaling, indicating that USP43 is a powerful suppressor of breast carcinogenesis. Future investigations will determine whether and how the EGFR/PI3K/AKT-USP43 reciprocally inhibitory loop functions in normal development, as well as in the development and progression of tumors originated from other tissues.

In summary, we report in the current study that USP43 is physically associated with the NuRD complex and functionally coordinates $\mathrm{H} 2 \mathrm{BK} 120$ deubiquitination and histone deacetylation to repress downstream target genes including EGFR and to suppress breast carcinogenesis. USP43 also exists in the cytoplasm where it is phosphorylated by AKT, leading to its binding to $14-3-3 \beta / \varepsilon$ heterodimer and cytoplasmic sequestration. Our study identified USP43 as a histone deubiquitinase and a potent suppressor of breast carcinogenesis. We propose the existence of a reciprocally inhibitory loop between the EGFR/PI3K/AKT signaling and USP43, whose imbalance drives breast carcinogenesis.

\section{MATERIALS AND METHODS}

Antibodies and reagents

Antibodies used: anti-FLAG, anti-tubulin, anti-fibronectin (Sigma); anti-USP43 (Abgent); anti-Mi-2 $\beta$, anti-MTA3, anti-HDAC1, anti-

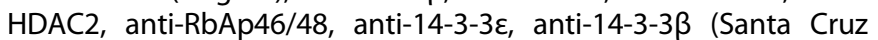
Biotechnology); anti-MBD3, anti-H2BK120ub, anti-H2AK119ub, anti-Phospho-(Ser/Thr), anti-E-cadherin, anti- $\gamma$-catenin, anti-Ncadherin (Cell Signaling Technology); anti-p-AKT (pSer473) (Servicebio); anti-H2B, anti-H2A (Merck Millipore); anti-H3, anti-EGFR (Abcam); anti-H3ac (Active Motif). Protein A/G Sepharose CL-4B beads were from Amersham Biosciences, and protease inhibitor cocktail was from Roche Applied Science.

Immunopurification and mass spectrometry

MCF-7 or HEK293T cells stably expressing FLAG-USP43 were washed twice with cold phosphate-buffered saline (PBS), scraped, and collected by centrifugation at $800 \times g$ for $5 \mathrm{~min}$. Cellular extracts were prepared by lysing the cells in lysis buffer containing protease inhibitor cocktail (Roche) and phosphatase inhibitor (Applygen). Anti-FLAG immunoaffinity resin (Sigma) was prepared according to the manufacturer's protocol. Cell lysates were applied to the immunoaffinity resin to enable adsorption of the protein complex. After binding, the resin was washed with cold PBS plus $0.3 \%$ Nonidet P-40. FLAG peptide (Sigma) was applied to the resin to elute the FLAG-tagged protein-associated complex as described by the vendor. The eluates were collected and resolved 
on NuPAGE 4-12\% Bis-Tris gel (Invitrogen), silver-stained (Pierce) and subjected to LC-MS/MS sequencing and data analysis.

Fast protein liquid chromatography

Cellular extracts were prepared by lysing the cells in lysis buffer containing protease inhibitor and phosphatase inhibitor. Approximately $5 \mathrm{mg}$ protein was concentrated to $0.5 \mathrm{~mL}$ using a Millipore Ultra free centrifugal filter apparatus ( $3 \mathrm{kDa}$ nominal molecular mass limit), and then applied to an $850 \times 20 \mathrm{~mm}$ Superose 6 size exclusion column (Amersham Biosciences) that had been equilibrated with buffer $\mathrm{D}$ containing $1 \mathrm{mM}$ dithiothreitol and calibrated with protein standards (blue dextran, $2000 \mathrm{kDa}$; thyroglobulin, 669 $\mathrm{kDa}$; ferritin, $440 \mathrm{kDa}$; catalase, $232 \mathrm{kDa}$; bovine serum albumin, 67 $\mathrm{kDa}$; and RNase A, $13.7 \mathrm{kDa}$, all from Amersham Biosciences). Elution was carried out at a flow rate of $0.5 \mathrm{~mL} / \mathrm{min}$ and fractions collected.

SILAC labeling and quantitative proteomics analysis

MCF-7 cells were grown in Dulbecco's modified Eagle's medium supplemented with $10 \%$ fetal bovine serum and either the "light" form of $\left[{ }^{12} \mathrm{C}_{6}\right]$-L-lysine or "heavy" $\left[{ }^{13} \mathrm{C}_{6}\right]$-L-lysine for more than six generations to achieve $>97 \%$ labeling efficiency. After that, the cells were further expanded in SILAC media to the desired cell number $\left(\sim 5 \times 10^{8}\right)$ in $15 \times 150 \mathrm{~mm}^{2}$ flasks. Cells infected with lentivirus vector or vectors carrying USP43 expression constructs were then collected and the histones were acid extracted. The histones from the "heavy" and "light" form cells were mixed 1:1 by protein content. Proteins were digested sequentially with trypsin followed by peptide desalting. Peptides were IP with anti-di-Gly antibody-conjugated agarose beads in the PTMScan ubiquitin remnant motif (K- $\varepsilon-\mathrm{GG})$ kit (Cell Signaling Technology) for $2 \mathrm{~h}$ at $4{ }^{\circ} \mathrm{C}$. The eluted peptides were cleaned with C18 ZipTips (Millipore) according to the manufacturer's instructions, followed by analysis with LC-MS/MS. The resulting MS/ MS data were processed using MaxQuant with integrated Andromeda search engine (version 1.4.1.2).

High-content screening

HeLa cells were transfected with siRNA pools against DUBs and immunofluorescently stained with antibodies against H2BK120ub. The level of H2BK120ub was analyzed by immunofluorescent staining followed by HCS microscopy. The experiments were biologically repeated three times and each time the experiments were performed in triplicate for each DUB.

Preparation of mononucleosomes

HeLa cells were collected by cold PBS, resuspended in lysis buffer $(10 \mathrm{mM} \mathrm{NaCl}, 3 \mathrm{mM} \mathrm{MgCl} 2,0.4 \%$ Nonidet P-40 and $10 \mathrm{mM}$ Tris- $\mathrm{HCl}$, $\mathrm{pH}$ 7.5) in the presence of protease inhibitor and the nuclei were pelleted. Glycerol buffer (0.1 mM EDTA, $5 \mathrm{mM} \mathrm{MgAc} 2,25 \%$ (vol/ vol) glycerol and $10 \mathrm{mM}$ Tris- $\mathrm{HCl}, \mathrm{pH} 7.4$ ) was added to give a final concentration of $1-2 \mathrm{mg} / \mathrm{mL}$ nuclei. To generate nucleosomal material, digestions were conducted by adding 1 volume of $2 \times$ micrococcal nuclease (MNase) buffer $(50 \mathrm{mM} \mathrm{KCl}, 8 \mathrm{mM} \mathrm{MgCl}$, $2 \mathrm{mM} \mathrm{CaCl}_{2}$ and $100 \mathrm{mM}$ Tris- $\mathrm{HCl}, \mathrm{pH}$ 7.4) and $3000 \mathrm{U} / \mathrm{mL}$ MNase. The reaction was incubated for $15 \mathrm{~min}$ at $37^{\circ} \mathrm{C}$ and stopped by adding EDTA to a final concentration of $10 \mathrm{mM}$. The mononucleosomes were then purified on sucrose gradients. ${ }^{50,58}$

Histone deubiquitination and histone deacetylation assays Calf thymus bulk histones (Sigma) or mononucleosomes were incubated with FLAG-USP43 or FLAG-MTA3 IPs at $37^{\circ} \mathrm{C}$ for $90 \mathrm{~min}$ in deubiquitination assay buffer $(50 \mathrm{mM} \mathrm{NaCl}, 1 \mathrm{mM}$ DTT and 50 $\mathrm{mM}$ Tirs- $\mathrm{HCl}, \mathrm{pH} 7.5)$ or histone deacetylation assay buffer (150 $\mathrm{mM} \mathrm{NaCl}, 1 \mathrm{mM}$ DTT and $10 \mathrm{mM}$ Tirs- $\mathrm{HCl}, \mathrm{pH} 8.0$ ). Each reaction was stopped by mixing with sodium dodecyl sulfate (SDS) sample loading buffer followed by western blot analysis with antibodies against H2BK120ub, H3ac, H2B or H3.
qChIP, ChIP and Re-ChIP

MCF-7 cells were crosslinked using $1 \%$ formaldehyde for $10 \mathrm{~min}$ at room temperature and quenched by the addition of glycine to a final concentration of $125 \mathrm{mM}$ for $5 \mathrm{~min}$. The fixed cells were resuspended in lysis buffer ( $1 \%$ SDS, $5 \mathrm{mM}$ EDTA and $50 \mathrm{mM}$ Tris$\mathrm{HCl}, \mathrm{pH} 8.1$ ) in the presence of protease inhibitors, then subjected to 30 cycles (30-s on and off) of sonication (Bioruptor, Diagenode) to generate chromatin fragments of $\sim 300 \mathrm{bp}$ in length. Lysates were diluted in buffer containing 1\% Triton X-100, 2 mM EDTA, $150 \mathrm{mM} \mathrm{NaCl}, 20 \mathrm{mM}$ Tris- $\mathrm{HCl}(\mathrm{pH} 8.1)$ and protease inhibitors. For $\mathrm{IP}$, the diluted chromatin was incubated with normal lgG (control) or specific antibodies for $12 \mathrm{~h}$ at $4{ }^{\circ} \mathrm{C}$ with constant rotation, $50 \mu \mathrm{L}$ of $50 \%(\mathrm{vol} / \mathrm{vol})$ protein A/G Sepharose beads were then added, and the incubation was continued for an additional $2 \mathrm{~h}$. Beads were washed with the following buffers: TSE I (0.1\% SDS, $1 \%$ Triton $\mathrm{X}-100,2 \mathrm{mM}$ EDTA, $150 \mathrm{mM} \mathrm{NaCl}$ and $20 \mathrm{mM}$ Tris- $\mathrm{HCl}$, pH 8.0); TSE II $(0.1 \%$ SDS, $1 \%$ Triton X-100, $2 \mathrm{mM}$ EDTA, $500 \mathrm{mM} \mathrm{NaCl}$ and 20 $\mathrm{mM}$ Tris- $\mathrm{HCl}, \mathrm{pH} 8.0)$; TSE III (0.25 M LiCl, 1\% Nonidet P-40, $1 \%$ sodium deoxycholate, $1 \mathrm{mM}$ EDTA and $10 \mathrm{mM}$ Tris- $\mathrm{HCl}, \mathrm{pH} 8.0$ ) and TE (1 mM EDTA and $10 \mathrm{mM}$ Tris- $\mathrm{HCl}, \mathrm{pH} 8.0)$. The pulled-down chromatin complex and input were de-crosslinked at $55^{\circ} \mathrm{C}$ for $12 \mathrm{~h}$ in elution buffer $\left(1 \% \mathrm{SDS} \text { and } 0.1 \mathrm{M} \mathrm{NaHCO}_{3}\right)^{59}$ The eluted DNA was purified with the QIAquick PCR Purification Kit. qChIPs were performed using Power SYBR Green PCR Master Mix and ABI PRISM 7500 sequence detection system (Applied Biosystems, Foster City, CA). Re-ChIPs were carried out in essentially the same way as primary IPs. Bead eluates from the first IP were incubated with $10 \mathrm{mM}$ DTT at $37^{\circ} \mathrm{C}$ for $30 \mathrm{~min}$ and diluted 1:50 in dilution buffer (1\% Triton X-100, $2 \mathrm{mM}$ EDTA, $150 \mathrm{mM} \mathrm{NaCl}$ and $20 \mathrm{mM}$ Tris- $\mathrm{HCl}, \mathrm{pH} 8.0)$ followed by re-IP with the second antibodies. The final elution step was performed using 1\% SDS solution in TrisEDTA buffer, $\mathrm{pH}$ 8.0. The sequences of the primers used are listed as following:

\begin{tabular}{|c|c|c|}
\hline EGFR & $\mathrm{F}$ & 5'-CACAAGTGGAAGCCCTCTCA-3' \\
\hline EGFR & $\mathrm{R}$ & 5'-CGTGGCAGCCTCAATGATGT-3' \\
\hline MAD1L1 & $\mathrm{F}$ & 5'-CTTGGAAGTGGCCGGGTTAT-3' \\
\hline MAD1L1 & $\mathrm{R}$ & 5'-AGGGATGAGTCCTAACGGCT-3' \\
\hline TNC & $\mathrm{F}$ & 5'-TGGGTCCGTTTTTTGGGGAAA-3' \\
\hline TNC & $\mathrm{R}$ & 5'-AGCCCGATCCACATCACATC-3' \\
\hline NTF3 & $\mathrm{F}$ & 5'-CAGGCTCTCCAGCCAGTAAG-3' \\
\hline NTF3 & $\mathrm{R}$ & 5'-TTCTGAATTTGCTGGGGGCA-3' \\
\hline BUB3 & $\mathrm{F}$ & 5'-ACACATCTGCCGACACTAGC-3' \\
\hline BUB3 & $\mathrm{R}$ & 5'-GCAGTTCTTTGGACGCAGTT-3' \\
\hline$A B L 1$ & $\mathrm{~F}$ & 5'-CCTTGCTGTCAAGGGGTCTC-3' \\
\hline$A B L 1$ & $\mathrm{R}$ & 5'-GCCCTCGACTCTGACGAAAA-3' \\
\hline COL27A1 & $\mathrm{F}$ & 5'-GGATGCGTTTCGTGTGAACC-3' \\
\hline COL27A1 & $\mathrm{R}$ & 5'-CTACCACCTCTTGCCACTCG-3' \\
\hline PHLPP1 & $\mathrm{F}$ & 5'-GCCGATCCCCTTACGAGAAC-3' \\
\hline PHLPP1 & $\mathrm{R}$ & 5'-GCGGAGCTTCAGGGTAAACA-3' \\
\hline CAMK2B & $\mathrm{F}$ & 5'-GCCCAGTGGTCTAAAGTCCC-3' \\
\hline CAMK2B & $\mathrm{R}$ & 5'-TCTCCTTTTCCAGCACGGTC-3' \\
\hline CALM2 & $\mathrm{F}$ & 5'-CCACGATAACAAGGGGCCAT-3' \\
\hline CALM2 & $\mathrm{R}$ & 5'-ATCTGGACACGGGCAAATGT-3' \\
\hline ATG12 & $\mathrm{F}$ & 5'-GCTGGATACTCACGTAGGGC-3' \\
\hline ATG12 & $\mathrm{R}$ & 5'-GTCGCGTGCGGGAGG-3' \\
\hline FYN & $\mathrm{F}$ & 5'-CCGATGCACTCCCAACTGTA-3' \\
\hline FYN & $\mathrm{R}$ & 5'-CGGCGACTAAGGAACCTGAA-3' \\
\hline RALA & $\mathrm{F}$ & 5'-AAAGAACTCGCAGACCGTCC-3' \\
\hline RALA & $\mathrm{R}$ & 5'-GGGGTATTCAGAGCGGAGC-3' \\
\hline
\end{tabular}


Statistical analysis

Results were reported as mean \pm SD for triplicate experiments unless otherwise indicated. SPSS V.17.0 and two-tailed unpaired $t$ test were used for statistical analysis. The correlation coefficients were calculated by $\mathrm{R}$ programming. Breast tumor datasets were downloaded from http://www.ncbi.nlm.nih.gov/geo. Data for Kaplan-Meier survival analysis were from http://kmplot.com/ analysis/index.php? $\mathrm{p}=$ service $\&$ cancer $=$ breast.

\section{ACKNOWLEDGEMENTS}

This work was supported by grants (91219201 and 81530073 to Y.S., and 81372223 , 81422034 and 31571340 to L.S.) from the National Natural Science Foundation of China, and grants (2016YFC1302304 to Y.S. and 2014CB542004 to L.S.) from the Ministry of Science and Technology of China.

\section{AUTHOR CONTRIBUTIONS}

L.H., Luyang S. and Y.S. conceived the project and designed the experiments; L.H., Xinhua L. and J.Y. performed experiments and analyzed data; F.P. performed pathologic analysis; J.Y., Z.Y. and L.L. performed animal experiments; W.L., S.L., Xujun L., J.R., Y.W., Lin S., C.G., Y.Z., Xiaohan Y., Xia Y., J.L. and R.L. provided technical assistance; L.H., Luyang S. and Y.S. wrote the manuscript.

\section{ADDITIONAL INFORMATION}

Supplementary information accompanies this paper at https://doi.org/10.1038/ s41422-018-0079-6.

Competing interests: The authors declare no competing interests.

\section{REFERENCES}

1. Appert-Collin, A., Hubert, P., Cremel, G. \& Bennasroune, A. Role of ErbB receptors in cancer cell migration and invasion. Front. Pharmacol. 6, 283 (2015).

2. Chan, S. K., Hill, M. E. \& Gullick, W. J. The role of the epidermal growth factor receptor in breast cancer. J. Mammary Gland Biol. Neoplasia 11, 3-11 (2006).

3. Holbro, T., Civenni, G. \& Hynes, N. E. The ErbB receptors and their role in cancer progression. Exp. Cell Res. 284, 99-110 (2003).

4. Alanazi, I. O. \& Khan, Z. Understanding EGFR signaling in breast cancer and breast cancer stem cells: overexpression and therapeutic implications. Asian Pac. J. Cancer Prev. 17, 445-453 (2016).

5. Engelman, J. A., Luo, J. \& Cantley, L. C. The evolution of phosphatidylinositol 3kinases as regulators of growth and metabolism. Nat. Rev. Genet. 7, 606-619 (2006).

6. Engelman, J. A. Targeting PI3K signalling in cancer: opportunities, challenges and limitations. Nat. Rev. Cancer 9, 550-562 (2009).

7. Banfic, H. et al. A novel integrin-activated pathway forms PKB/Akt-stimulatory phosphatidylinositol 3,4-bisphosphate via phosphatidylinositol 3-phosphate in platelets. J. Biol. Chem. 273, 13-16 (1998).

8. Davies, M. A. et al. Regulation of Akt/PKB activity, cellular growth, and apoptosis in prostate carcinoma cells by MMAC/PTEN. Cancer Res. 59, 2551-2556 (1999).

9. Dheeraj, A. et al. Silibinin treatment inhibits the growth of hedgehog inhibitorresistant basal cell carcinoma cells via targeting EGFR-MAPK-Akt and hedgehog signaling. Photochem. Photobiol. 93, 999-1007 (2017).

10. Chakravarti, A. et al. The prognostic significance of phosphatidylinositol 3-kinase pathway activation in human gliomas. J. Clin. Oncol. 22, 1926-1933 (2004).

11. Wu, X. et al. Activation of diverse signalling pathways by oncogenic PIK3CA mutations. Nat. Commun. 5, 4961 (2014).

12. Stambolic, V. et al. Negative regulation of PKB/Akt-dependent cell survival by the tumor suppressor PTEN. Cell 95, 29-39 (1998).

13. Davis, N. M. et al. Deregulation of the EGFR/PI3K/PTEN/Akt/mTORC1 pathway in breast cancer: possibilities for therapeutic intervention. Oncotarget 5, 4603-4650 (2014).

14. Sun, M. et al. AKT1/PKBalpha kinase is frequently elevated in human cancers and its constitutive activation is required for oncogenic transformation in NIH3T3 cells. Am. J. Pathol. 159, 431-437 (2001).

15. Wilkinson, K. D. Regulation of ubiquitin-dependent processes by deubiquitinating enzymes. FASEB J. 11, 1245-1256 (1997).

16. Nijman, S. M. et al. A genomic and functional inventory of deubiquitinating enzymes. Cell 123, 773-786 (2005).
17. Sowa, M. E., Bennett, E. J., Gygi, S. P. \& Harper, J. W. Defining the human deubiquitinating enzyme interaction landscape. Cell 138, 389-403 (2009).

18. Fuchs, G. et al. RNF20 and USP44 regulate stem cell differentiation by modulating H2B monoubiquitylation. Mol. Cell 46, 662-673 (2012).

19. Singh, N. \& Singh, A. B. Deubiquitinases and cancer: a snapshot. Crit. Rev. Oncol. Hematol. 103, 22-26 (2016).

20. Wang, Q. et al. Stabilization of histone demethylase PHF8 by USP7 promotes breast carcinogenesis. J. Clin. Invest. 126, 2205-2220 (2016).

21. Li, X. et al. USP9X regulates centrosome duplication and promotes breast carcinogenesis. Nat. Commun. 8, 14866 (2017).

22. Engel, K. et al. USP9X stabilizes XIAP to regulate mitotic cell death and chemoresistance in aggressive B-cell lymphoma. EMBO Mol. Med. 8, 851-862 (2016).

23. Li, Z. et al. Ubiquitination of a novel deubiquitinating enzyme requires direct binding to von Hippel-Lindau tumor suppressor protein. J. Biol. Chem. 277, 4656-4662 (2002).

24. Li, Z. et al. Identification of a deubiquitinating enzyme subfamily as substrates of the von Hippel-Lindau tumor suppressor. Biochem. Biophys. Res. Commun. 294, 700-709 (2002).

25. Weake, V. M. \& Workman, J. L. Histone ubiquitination: triggering gene activity. Mol. Cell 29, 653-663 (2008).

26. Wright, D. E., Wang, C. Y. \& Kao, C. F. Histone ubiquitylation and chromatin dynamics. Front Biosci. (Landmark Ed.) 17, 1051-1078 (2012).

27. Jaaskelainen, $\mathrm{T}$. et al. Histone H2B ubiquitin ligases RNF20 and RNF40 in androgen signaling and prostate cancer cell growth. Mol. Cell. Endocrinol. 350, 87-98 (2012).

28. Wang, E. et al. Histone H2B ubiquitin ligase RNF20 is required for MLL-rearranged leukemia. Proc. Natl. Acad. Sci. USA 110, 3901-3906 (2013).

29. Duan, Y. et al. Ubiquitin ligase RNF20/40 facilitates spindle assembly and promotes breast carcinogenesis through stabilizing motor protein Eg5. Nat. Commun. 7, 12648 (2016).

30. Zhu, B. et al. Monoubiquitination of human histone $\mathrm{H} 2 \mathrm{~B}$ : the factors involved and their roles in HOX gene regulation. Mol. Cell 20, 601-611 (2005).

31. Nicassio, F. et al. Human USP3 is a chromatin modifier required for S phase progression and genome stability. Curr. Biol. 17, 1972-1977 (2007).

32. Zhao, Y. et al. A TFTC/STAGA module mediates histone $\mathrm{H} 2 \mathrm{~A}$ and $\mathrm{H} 2 \mathrm{~B}$ deubiquitination, coactivates nuclear receptors, and counteracts heterochromatin silencing. Mol. Cell 29, 92-101 (2008).

33. Joo, $\mathrm{H}$. Y. et al. Regulation of histone $\mathrm{H} 2 \mathrm{~A}$ and $\mathrm{H} 2 \mathrm{~B}$ deubiquitination and Xenopus development by USP12 and USP46. J. Biol. Chem. 286, 7190-7201 (2011).

34. Zhang, Z. et al. USP49 deubiquitinates histone $\mathrm{H} 2 \mathrm{~B}$ and regulates cotranscriptional pre-mRNA splicing. Genes \& Dev. 27, 1581-1595 (2013).

35. Fuchs, G. \& Oren, M. Writing and reading H2B monoubiquitylation. Biochim. Biophys. Acta 1839, 694-701 (2014).

36. Yuan, L. et al. Deubiquitylase OTUD3 regulates PTEN stability and suppresses tumorigenesis. Nat. Cell Biol. 17, 1169-1181 (2015).

37. Pan, J. et al. USP37 directly deubiquitinates and stabilizes c-Myc in lung cancer. Oncogene 34, 3957-3967 (2015).

38. Liao, J. \& Omary, M. B. 14-3-3 Proteins associate with phosphorylated simple epithelial keratins during cell cycle progression and act as a solubility cofactor. J. Cell. Biol. 133, 345-357 (1996).

39. Zhang, Y., LeRoy, G., Seelig, H. P., Lane, W. S. \& Reinberg, D. The dermatomyositisspecific autoantigen $\mathrm{Mi} 2$ is a component of a complex containing histone deacetylase and nucleosome remodeling activities. Cell 95, 279-289 (1998).

40. Xue, Y. et al. NURD, a novel complex with both ATP-dependent chromatinremodeling and histone deacetylase activities. Mol. Cell 2, 851-861 (1998).

41. Denslow, S. A. \& Wade, P. A. The human Mi-2/NuRD complex and gene regulation. Oncogene 26, 5433-5438 (2007).

42. Lee-Kwon, W. et al. Constitutively active phosphatidylinositol 3-kinase and AKT are sufficient to stimulate the epithelial $\mathrm{Na}+/ \mathrm{H}+$ exchanger 3. J. Biol. Chem. 276, 31296-31304 (2001).

43. Lindsley, C. W. The Akt/PKB family of protein kinases: a review of small molecule inhibitors and progress towards target validation: a 2009 update. Curr. Top. Med. Chem. 10, 458-477 (2010).

44. Si, W. et al. Dysfunction of the reciprocal feedback loop between GATA3- and ZEB2-nucleated repression programs contributes to breast cancer metastasis. Cancer Cell. 27, 822-836 (2015).

45. Hanahan, D. \& Weinberg, R. A. Hallmarks of cancer: the next generation. Cell 144, 646-674 (2011)

46. Lee, J. H., Kavanagh, J. J., Wildrick, D. M., Wharton, J. T. \& Blick, M. Frequent loss of heterozygosity on chromosomes $6 \mathrm{q}, 11$, and 17 in human ovarian carcinomas. Cancer Res. 50, 2724-2728 (1990).

47. Russell, S. E., Hickey, G. I., Lowry, W. S., White, P. \& Atkinson, R. J. Allele loss from chromosome 17 in ovarian cancer. Oncogene 5, 1581-1583 (1990). 
48. Liu, Y. et al. Deletions linked to TP53 loss drive cancer through p53-independent mechanisms. Nature 531, 471-475 (2016).

49. Wang, Y. et al. LSD1 is a subunit of the NuRD complex and targets the metastasis programs in breast cancer. Cell 138, 660-672 (2009).

50. Li, Q. et al. Binding of the JmjC demethylase JARID1B to LSD1/NuRD suppresses angiogenesis and metastasis in breast cancer cells by repressing chemokine CCL14. Cancer Res. 71, 6899-6908 (2011).

51. Fujita, N. et al. MTA3, a Mi-2/NuRD complex subunit, regulates an invasive growth pathway in breast cancer. Cell 113, 207-219 (2003).

52. Li, D. Q., Pakala, S. B., Nair, S. S., Eswaran, J. \& Kumar, R. Metastasis-associated protein $1 /$ nucleosome remodeling and histone deacetylase complex in cancer. Cancer Res. 72, 387-394 (2012).

53. Lai, A. Y. \& Wade, P. A. Cancer biology and NuRD: a multifaceted chromatin remodelling complex. Nat. Rev. Cancer 11, 588-596 (2011).
54. Shan, L. et al. FOXK2 elicits massive transcription repression and suppresses the hypoxic response and breast cancer carcinogenesis. Cancer Cell. 30, 708-722 (2016).

55. Bowen, N. J., Fujita, N., Kajita, M. \& Wade, P. A. Mi-2/NuRD: multiple complexes for many purposes. Biochim. Biophys. Acta 1677, 52-57 (2004).

56. Vergara, D. et al. Resveratrol inhibits the epidermal growth factor-induced epithelial mesenchymal transition in MCF-7 cells. Cancer Lett. 310, 1-8 (2011).

57. Guerrero-Zotano, A., Mayer, I. A. \& Arteaga, C. L. PI3K/AKT/mTOR: role in breast cancer progression, drug resistance, and treatment. Cancer Metastas. Rev. 35 515-524 (2016).

58. Shi, L. et al. Histone demethylase JMJD2B coordinates H3K4/H3K9 methylation and promotes hormonally responsive breast carcinogenesis. Proc. Natl. Acad. Sci. USA 108, 7541-7546 (2011).

59. Li, L. et al. SIRT7 is a histone desuccinylase that functionally links to chromatin compaction and genome stability. Nat. Commun. 7, 12235 (2016). 